\title{
Banach algebras with unique uniform norm II
}

by

\author{
S. J. Bhatt and H. V. Dedania (Vallabh Vidyanagar)
}

\begin{abstract}
Semisimple commutative Banach algebras $\mathcal{A}$ admitting exactly one uniform norm (not necessarily complete) are investigated. $\mathcal{A}$ has this Unique Uniform Norm Property iff the completion $U(\mathcal{A})$ of $\mathcal{A}$ in the spectral radius $r(\cdot)$ has UUNP and, for any non-zero spectral synthesis ideal $\mathcal{I}$ of $U(\mathcal{A}), \mathcal{I} \cap \mathcal{A}$ is non-zero. $\mathcal{A}$ is regular iff $U(\mathcal{A})$ is regular and, for any spectral synthesis ideal $\mathcal{I}$ of $\mathcal{A}, \mathcal{A} / \mathcal{I}$ has UUNP iff $U(\mathcal{A})$ is regular and for any spectral synthesis ideal $\mathcal{I}$ of $U(\mathcal{A}), \mathcal{I}=k(h(\mathcal{A} \cap \mathcal{I})$ ) (hulls and kernels in $U(\mathcal{A})) . \mathcal{A}$ has UUNP and the Shilov boundary coincides with the Gelfand space iff $\mathcal{A}$ is weakly regular in the sense that, given a proper, closed subset $F$ of the Gelfand space, there exists a non-zero $x$ in $\mathcal{A}$ having its Gelfand transform vanishing on $F$. Several classes of Banach algebras that are weakly regular but not regular, as well as those that are not weakly regular but have UUNP are exhibited. The UUNP is investigated for quotients, tensor products, and multiplier algebras. The property UUNP compares with the unique $C^{*}$-norm property on (not necessarily commutative) Banach *-algebras. The results are applied to multivariate holomorphic function algebras as well as to the measure algebra of a locally compact abelian group $G$. For a continuous weight $\omega$ on $G$, the Beurling algebra $L^{1}(G, \omega)$ (assumed semisimple) has UUNP iff it is regular.
\end{abstract}

1. Introduction. A uniform norm on a normed algebra $(\mathcal{A},\|\cdot\|)$ is a (not necessarily complete) submultiplicative norm $|\cdot|$ satisfying the square property $\left|x^{2}\right|=|x|^{2}(x \in \mathcal{A})$. In fact, in the presence of the square property, submultiplicativity is automatic [De]. If $|\cdot|$ is a uniform norm on $\mathcal{A}$, then $\mathcal{A}$ is semisimple and commutative; further, if $(\mathcal{A},\|\cdot\|)$ is complete, then the spectral radius $r(\cdot)=r_{\mathcal{A}}(\cdot)$ is (in fact, the greatest) uniform norm. $\mathcal{A}$ has UUNP (unique uniform norm property) if $\mathcal{A}$ admits exactly one uniform norm. Notice that any two equivalent uniform norms are equal. This aesthetically pleasing property arises naturally in [BhDe1] in the investigations of incomplete algebra norms on Banach algebras [TY] and spectral extension properties [Me1], and it is a natural companion of the celebrated unique

2000 Mathematics Subject Classification: Primary 46J05, 46J40, 46K99; Secondary 43A10, 43A20, 46J10.

Key words and phrases: unique uniform norm property, regular Banach algebras, unique $C^{*}$-norm property, multipliers, tensor product, Beurling algebras, multivariate holomorphic function algebras, measure algebras. 
complete norm property in Banach algebras ([BaDa], $[\mathrm{DL}])$, as well as the unique $C^{*}$-norm property $\left(\mathrm{U} C^{*} \mathrm{NP}\right)$ in Banach ${ }^{*}$-algebras $([\mathrm{Ba}],[\mathrm{HKV}])$. The present paper is a third paper in continuation of [BhDe1], [BhDe2]; however, it is independent of them.

The first purpose of the present paper is to place UUNP in proper perspective in the general theory of commutative Banach algebras. Our approach to UUNP exhibits the role of the enveloping uniform algebra $U(\mathcal{A})$ (which is the Hausdorff completion of $\mathcal{A}$ in the spectral radius) in understanding $\mathcal{A}$. In Section 2 , we show that $\mathcal{A}$ has UUNP iff $U(\mathcal{A})$ has UUNP and for any non-zero spectral synthesis ideal $\mathcal{I}$ of $U(\mathcal{A}), \mathcal{I} \cap \mathcal{A}$ is non-zero. We discuss a simple example of $\mathcal{A}$ such that $U(\mathcal{A})$ has UUNP, but $\mathcal{A}$ does not have UUNP. We also show that, if $\mathcal{A}$ has UUNP and if $\mathcal{I}$ is a spectral synthesis ideal of $\mathcal{A}$, then $\mathcal{I}$ has UUNP, but $\mathcal{A} / \mathcal{I}$ fails to have UUNP. The property UUNP turns out to be closely related with regularity. A Banach algebra $(\mathcal{A},\|\cdot\|)$ (assumed semisimple and commutative) is regular (called completely regular in [BD]) if, given a closed subset $F$ of the Gelfand space $\Delta(\mathcal{A})$ and $\varphi \in \Delta(\mathcal{A}) \backslash F$, there exists $x \in \mathcal{A}$ such that $\widehat{x}(F)=\{0\}$, $\widehat{x}(\varphi) \neq 0$. Regularity implies UUNP but not conversely [BhDe1]. The relevance of UUNP to regularity is revealed by Theorem 2.6, which is one of the main results of Section 2. It states that $\mathcal{A}$ is regular iff $U(\mathcal{A})$ is regular and $\mathcal{A} / \mathcal{I}$ has UUNP for any spectral synthesis ideal $\mathcal{I}$ of $\mathcal{A}$ iff $U(\mathcal{A})$ is regular and $\mathcal{I}=k(h(\mathcal{A} \cap \mathcal{I})$ ) (the kernel and hull in $U(\mathcal{A})$ ) for any spectral synthesis ideal $\mathcal{I}$ of $U(\mathcal{A})$. Following [BhDe1], $\mathcal{A}$ is weakly regular if given a proper closed set $F$ in $\Delta(\mathcal{A})$, there exists $x \neq 0$ in $\mathcal{A}$ such that $\widehat{x}(F)=\{0\}$. This notion seems to be of independent interest. We give a fairly general construction of Banach algebras which are weakly regular, but not regular. It so happens that $\mathcal{A}$ is weakly regular iff $\Delta(\mathcal{A})=\partial(\mathcal{A})$ (Shilov boundary) and $\mathcal{A}$ has UUNP. This is used to discuss conditions under which regularity is equivalent to UUNP. $\mathcal{A}$ has $S E P$ (spectral extension property) [Me1] if for any (submultiplicative) norm $|\cdot|$ on $\mathcal{A}, r(x) \leq|x|(x \in \mathcal{A})$. The following graph summarizes the inter-relations among these four properties.

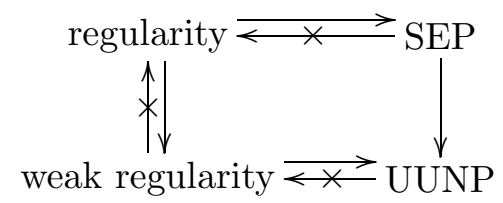

Does UUNP imply SEP? Our conjecture is that it does not.

The second purpose of the paper is to compare a commutative (not necessarily involutive) Banach algebra $\mathcal{A}$ having UUNP with a (not necessarily commutative) Banach ${ }^{*}$-algebra $\mathcal{B}$ having $\mathrm{U} C^{*} \mathrm{NP}$. This is motivated by the apparent similarity between the $C^{*}$-property $\left\|x^{*} x\right\|=\|x\|^{2}$ of a $C^{*}$-norm and the square property $\left\|x^{2}\right\|=\|x\|^{2}$ of a uniform norm. Then $U(\mathcal{A})$ corre- 
sponds to $C^{*}(\mathcal{B})$, the enveloping $C^{*}$-algebra of $\mathcal{B}$; and regularity corresponds to ${ }^{*}$-regularity $[\mathrm{Ba}],[\mathrm{P}]$. Though our results for $\mathcal{A}$ relating regularity and UUNP are comparable to the results in $[\mathrm{Ba}]$ for $\mathcal{B}$ relating *-regularity and $\mathrm{U} C^{*} \mathrm{NP}$, the present paper also exhibits that both these aspects are not completely analogous. This is expected in view of the facts that though $C^{*}(\mathcal{B})$ has $C^{*} \mathrm{NP}$ and is *-regular, $U(\mathcal{A})$ need neither have UUNP nor be regular; on the other hand, $\mathcal{A}$ is inverse closed in $U(\mathcal{A})$, while $\mathcal{B}$ need not be inverse closed in $C^{*}(\mathcal{B})$, unless it is hermitian. It is shown in Section 3 that the algebraic tensor product $\mathcal{A} \otimes \mathcal{B}$ has UUNP iff both $\mathcal{A}$ and $\mathcal{B}$ have UUNP. This supplements Tomiyama's result that $\mathcal{A} \widehat{\otimes}_{\alpha} \mathcal{B}$ (completion in a cross norm $\alpha$ ) is regular iff both $\mathcal{A}$ and $\mathcal{B}$ are regular; on the other hand, it compares with the result in $[\mathrm{HKV}]$ that if $\mathcal{A}$ and $\mathcal{B}$ are Banach ${ }^{*}$-algebras, then $\mathcal{A} \otimes \mathcal{B}$ is *-regular (resp. has $\mathrm{U} C^{*} \mathrm{NP}$ ) iff each of $\mathcal{A}, \mathcal{B}$ and $C^{*}(\mathcal{A}) \otimes C^{*}(\mathcal{B})$ is *-regular (resp. has $\left.\mathrm{U}^{*} \mathrm{NP}\right)$. In [BhDe2], it is shown that unlike $\mathrm{U}^{*} \mathrm{NP}$ [Ba, p. 849], $\mathcal{A}$ has UUNP iff the unitization $\mathcal{A}_{e}$ has UUNP. This throws further light on the MEtatheorem envisaged in [Bh1], [Bh2] that there exists a structural analogy between certain (and not all) aspects of $C^{*}$-algebras and uniform Banach algebras ( $u B$-algebras); more generally, between hermitian Banach *-algebras and Banach algebras commutative modulo the radical. This also implies that if $\mathcal{A}$ has UUNP, then each of the $\mathcal{A}$-valued function algebras $C_{0}(X, \mathcal{A}), C^{n}([a, b], \mathcal{A}), \operatorname{lip}_{\alpha}([a, b], \mathcal{A}), L^{1}(G, \mathcal{A}), L^{p}(G, \mathcal{A})$ ( $G$ a compact abelian group, $1<p<\infty$ ) has UUNP.

In Section 4, we investigate the UUNP for the Beurling algebras $L^{1}(G, \omega)$. It is shown that if $L^{1}(G, \omega)$ is semisimple, then it has UUNP iff it is regular. If $H$ is a subgroup of the rationals $\mathbb{Q}$ containing 1 , and if $\omega$ is a weight on $H$, then $\ell^{1}(H, \omega)$ has UUNP iff $\ell^{1}(\mathbb{Z}, \omega)$ has UUNP. It is shown that the semigroup algebras $L^{1}\left(\mathbb{R}^{+}, \omega\right)$ and $\ell^{1}\left(\mathbb{Z}^{+}, \omega\right)$ do not have UUNP for all weights $\omega$.

In the brief Section 5, we investigate sufficient conditions under which a $u B$-algebra has UUNP. This leads to the constructions of the variants of the polydisc algebra and the ball algebra in $\mathbb{C}^{n}$, as well as those of the $H^{\infty}$-algebras on the polydisc and the ball which have UUNP, but are not weakly regular. Note that the polydisc algebra and the ball algebra in $\mathbb{C}^{n}$, as well as their $H^{\infty}$-analogues, do not have UUNP.

In Section 6, we investigate UUNP for the multiplier algebra $M(\mathcal{A})$ of $\mathcal{A}$. If $M(\mathcal{A})$ has UUNP, then $\mathcal{A}$ has UUNP; and the converse holds if $\mathcal{A}$ is a $u B$-algebra. In the case where $\mathcal{A}$ is weakly regular, the closed ideal $M_{00}(\mathcal{A})=\{T \in M(\mathcal{A}): \widehat{T}=0$ on $h(\mathcal{A})\}$ has UUNP; while the closed ideal $M_{0}(\mathcal{A})=\left\{T \in M(\mathcal{A}):\left.\widehat{T}\right|_{\Delta(\mathcal{A})} \in C_{0}(\Delta(\mathcal{A}))\right\}$ fails to have UUNP. When $\mathcal{A}$ is weakly regular, the closed subalgebra $\operatorname{Dec} M(\mathcal{A})$ consisting of multipliers defining decomposable operators on $M(\mathcal{A})$ by multiplication has UUNP; whereas the subalgebra $D M(\mathcal{A})$ consisting of decomposable multipliers on $\mathcal{A}$ 
fails to have UUNP. These results are applied to the measure algebra $M(G)$ of a locally compact abelian group $G$. Note that $M(G) \cong M\left(L^{1}(G)\right)$. Like regularity, $M(G)$ has UUNP iff $G$ is discrete [BhDe1]. In the non-discrete case, $M(G)$ admits minimum and maximum uniform norms. The Banach subalgebras of $M(G)$ which have UUNP include the algebra $L^{1}(G)$, the closed subalgebra generated by $\left\{L^{1}(G), M_{\mathrm{d}}(G)\right\}$, the algebra Dec $M(G)$ consisting of measures $\mu$ whose Fourier-Stieltjes transform $\widehat{\mu}$ are hull-kernel continuous on $\Delta(M(G))$, as well as the algebra $D M(G)$ consisting of measures defining decomposable convolution operators on $L^{1}(G)$. In fact, $\operatorname{Dec} M(G)$ and $D M(G)$ are weakly regular, non-regular, having hermitian involution and having UUNP.

2. Ideals, quotients, UUNP and regularity. Throughout the paper, let $(\mathcal{A},\|\cdot\|)$ be a semisimple commutative Banach algebra, not necessarily unital. Let $\Delta(\mathcal{A})$ be its Gelfand space. Let $U(\mathcal{A})$ be the $u B$-algebra obtained by completing $(\mathcal{A}, r(\cdot))$, where $r(x)=\sup \{|\varphi(x)|: \varphi \in \Delta(\mathcal{A})\}(x \in \mathcal{A})$, the spectral radius. An ideal $\mathcal{I}$ of $\mathcal{A}$ is a spectral synthesis ideal (or a semisimple ideal) if $\mathcal{I}=k(h(\mathcal{I}))$, the kernel of the hull of $\mathcal{I}$ in $\mathcal{A}$; equivalently, the quotient algebra $\mathcal{A} / \mathcal{I}$ is semisimple. A closed set $F \subset \Delta(\mathcal{A})$ is a set of uniqueness for a subalgebra $\mathcal{B}$ of $\mathcal{A}$ if $|x|_{F}=\sup \{|\varphi(x)|: \varphi \in F\} \quad(x \in \mathcal{B})$ defines a norm on the algebra $\mathcal{B}$.

It is shown in [Ba, Proposition 2.4] that a ${ }^{*}$-semisimple Banach *-algebra $B$ has $\mathrm{U} C^{*} \mathrm{NP}$ iff, for any non-zero closed ideal $\mathcal{I}$ of $C^{*}(\mathcal{B}), \mathcal{B} \cap \mathcal{I} \neq(0)$. The following compares with this.

Proposition 2.1. The following are equivalent:

(i) $\mathcal{A}$ has UUNP;

(ii) $U(\mathcal{A})$ has $U U N P$, and every closed subset $F$ of $\Delta(U(\mathcal{A}))$ which is a set of uniqueness for $\mathcal{A}$ is also a set of uniqueness for $U(\mathcal{A})$;

(iii) $U(\mathcal{A})$ has $U U N P$, and $\mathcal{I} \cap \mathcal{A} \neq(0)$ for any non-zero spectral synthesis ideal $\mathcal{I}$ of $U(\mathcal{A})$.

Proof. (i) $\Rightarrow($ ii). Clearly $U(\mathcal{A})$ has UUNP. Let $F \subset \Delta(U(\mathcal{A}))$ be a closed set of uniqueness for $\mathcal{A}$. Define $|x|_{F}=\sup \{|\varphi(x)|: \varphi \in F\}(x \in U(\mathcal{A}))$. Then $|\cdot|_{F}$ is a uniform seminorm on $U(\mathcal{A})$ which is a norm on $\mathcal{A}$. By $(\mathrm{i}),|\cdot|_{F}=r(\cdot)$ on $\mathcal{A}$. Since $\mathcal{A}$ is dense in $U(\mathcal{A}),|\cdot|_{F}=r(\cdot)$ on $U(\mathcal{A})$ too. Thus $F$ is a set of uniqueness for $U(\mathcal{A})$.

(ii) $\Rightarrow$ (iii). Let $\mathcal{I}$ be a non-zero spectral synthesis ideal of $U(\mathcal{A})$. Define $F=h(\mathcal{I})=\{\varphi \in \Delta(U(\mathcal{A})): \varphi(x)=0$ for all $x \in \mathcal{I}\}$. Then $F$ is not a set of uniqueness for $U(\mathcal{A})$. Hence, by (ii), $F$ is not a set of uniqueness for $\mathcal{A}$, and so $\mathcal{I} \cap \mathcal{A}=k(F) \cap \mathcal{A} \neq(0)$.

(iii) $\Rightarrow($ i). Let $|\cdot|$ be a uniform norm on $\mathcal{A}$. Then $|\cdot| \leq r(\cdot)$ on $\mathcal{A}$, and so $|\cdot|$ can be extended as a uniform seminorm on $U(\mathcal{A})$. Let $\mathcal{I}=\operatorname{ker}(|\cdot|)$. Then 
$\mathcal{I}$ is a spectral synthesis ideal of $U(\mathcal{A})$. Since $|\cdot|$ is a norm on $\mathcal{A}, \mathcal{I} \cap \mathcal{A}=(0)$. Hence $\mathcal{I}=(0)$ by (iii). Thus $|\cdot|$ is a norm on $U(\mathcal{A})$. Since $U(\mathcal{A})$ has UUNP, $|\cdot|=r(\cdot)$ on $U(\mathcal{A})$, and so on $\mathcal{A}$. Thus $\mathcal{A}$ has UUNP.

Example 2.2. There exists $\mathcal{A}$ not having UUNP, but $U(\mathcal{A})$ is regular. Let $\omega=\left(\omega_{n}\right)_{n=-\infty}^{\infty}$ be defined as $\omega_{n}=\exp \left(\alpha_{n}\right)$, where $\alpha_{0}=1$, and $\alpha_{n}=$ $|n| /\{\log (|n|)+1\}(n \in \mathbb{Z} \backslash\{0\})$. Let $\ell^{1}(\mathbb{Z}, \omega)=\left\{a=\left(a_{n}\right)_{n=-\infty}^{\infty}\right.$ : each $a_{n} \in \mathbb{C}$, and $\left.\sum_{n=-\infty}^{\infty}\left|a_{n}\right| \omega_{n}<\infty\right\}$, a convolution Banach ${ }^{*}$-algebra with the norm $\|a\|=\sum_{n=-\infty}^{\infty}\left|a_{n}\right| \omega_{n}$ and the involution $\left(a_{n}\right)^{*}=\left(\bar{a}_{-n}\right)$. Then $\ell^{1}(\mathbb{Z}, \omega)$ is a hermitian, semisimple, commutative Banach *-algebra with more than one $C^{*}$-norm [Ba, p. 847]. Now, in a commutative Banach *-algebra, a $C^{*}$-norm is a uniform norm. Thus $\mathcal{A}$ does not have UUNP. On the other hand, the hermiticity of $\mathcal{A}$ implies that $r(x)=m(x)(x \in \mathcal{A})$, where $m$ is the GelfandNaimark pseudo-norm [BD, Theorem 3, p. 188]. Hence $U(\mathcal{A})=C^{*}(\mathcal{A})$, which is regular.

The following compares with the well known fact that, if $\mathcal{I}$ is a closed ideal of $\mathcal{A}$ and if $\mathcal{A}$ is regular, then both $\mathcal{I}$ and $\mathcal{A} / \mathcal{I}$ are regular.

Proposition 2.3. Let $\mathcal{A}$ have UUNP and $\mathcal{I}$ be a closed ideal of $\mathcal{A}$. If $\mathcal{I}$ is a spectral synthesis ideal of $\mathcal{A}$, or if $\overline{\mathcal{I}}$ (closure in $U(\mathcal{A})$ ) is a spectral synthesis ideal of $U(\mathcal{A})$, then $\mathcal{I}$ has $U U N P$. The quotient algebra $\mathcal{A} / \mathcal{I}$ need not have UUNP.

Proof. (A) Note that $\Delta(\mathcal{I}) \cong \Delta(\mathcal{A}) \backslash h(\mathcal{I})$ and $\partial(\mathcal{I}) \subseteq \partial(\mathcal{A})$. Let $F$ be a closed subset of $\Delta(\mathcal{I})$ not containing $\partial(\mathcal{I})$. By [BhDe1, Theorem 2.3], it is sufficient to prove that $F$ is not a set of uniqueness for $\mathcal{I}$. Let $W=\Delta(\mathcal{I}) \backslash F$, and $K=\Delta(\mathcal{A}) \backslash W$. Since $\Delta(\mathcal{I})$ is open in $\Delta(\mathcal{A}), K$ is closed in $\Delta(\mathcal{A})$, and $K$ does not contain $\partial(\mathcal{A})$. Again by $[\mathrm{BhDe} 1$, Theorem 2.3] applied to $\mathcal{A}$, there exists $a \in \mathcal{A}, a \neq 0$, such that $\left.\widehat{a}\right|_{K}=0$. Fix $\varphi \in W, \varphi(a) \neq 0$. We may assume $\varphi(a)=1$. Since $\psi(a)=0$ for all $\psi \in h(\mathcal{I})$, we have $a \in k(h(\mathcal{I}))$.

CASE (i). Let $\mathcal{I}$ be a spectral synthesis ideal of $\mathcal{A}$. Then $k(h(\mathcal{I}))=I$. Thus $a \in \mathcal{I}$. Since $F \subseteq K$ and $\left.\widehat{a}\right|_{K}=0$, we have $\left.\widehat{a}\right|_{F}=0$. This shows that $F$ is not a set of uniqueness for $\mathcal{I}$.

CASE (ii). Assume that $\overline{\mathcal{I}}$ is a spectral synthesis ideal of $U(\mathcal{A})$. Noting that $\Delta(\mathcal{A})=\Delta(U(\mathcal{A}))$ and $h(\mathcal{I})=h(\overline{\mathcal{I}})$, we see that $a \in k(h(\mathcal{I})) \subset k(h(\overline{\mathcal{I}}))$ $=\overline{\mathcal{I}}$. Thus $a \in \overline{\mathcal{I}} \cap \mathcal{A}$. Choose $y \in \mathcal{I}$ such that $r(a-y) r(a)<1$. Then $y a \in \mathcal{I}$ and $\left.(y a)^{\wedge}\right|_{F}=0$. If $(y a)^{\wedge}(\varphi)=0$, then

$$
1=\left(a^{2}\right)^{\wedge}(\varphi)-(y a)^{\wedge}(\varphi)=\left|(a-y)^{\wedge}(\varphi) \widehat{a}(\varphi)\right| \leq r(a-y) r(a)<1,
$$

which is a contradiction. Thus $(y a)^{\wedge}(\varphi) \neq 0$, and so $F$ is not a closed set of uniqueness for $\mathcal{I}$.

(B) Let $\Delta_{r}=\{z \in \mathbb{C}:|z|<r\}$. For a bounded open set $U$, let $H(U)$ denote the algebra of all holomorphic functions on $U, C(\bar{U})$ denote the 
algebra of all continuous functions on $\bar{U}$. Let $\mathcal{A}=\left\{f \in C\left(\bar{\Delta}_{2}\right): f \in H\left(\Delta_{1}\right)\right\}$, a $u B$-algebra with pointwise operations and the norm $\|f\|_{\infty}=\sup \{|f(z)|$ : $\left.z \in \bar{\Delta}_{2}\right\}$. By [Me1, Example 2], $\mathcal{A}$ has SEP, and hence it has UUNP. Now $\mathcal{I}=\left\{f \in \mathcal{A}: f=0\right.$ on $\left.\bar{\Delta}_{1}\right\}$ is a spectral synthesis ideal of $\mathcal{A}$, and $\mathcal{A} / \mathcal{I}$ is isometrically isomorphic to the disc algebra $\mathcal{A}\left(\bar{\Delta}_{1}\right)=\left\{f \in C\left(\bar{\Delta}_{1}\right): f \in\right.$ $\left.H\left(\Delta_{1}\right)\right\}$, which obviously admits infinitely many distinct uniform norms.

Does there exist a spectral synthesis ideal $\mathcal{I}$ of $\mathcal{A}$ such that both $\mathcal{I}$ and $\mathcal{A} / \mathcal{I}$ have UUNP, but $\mathcal{A}$ does not have UUNP? Let $\mathcal{I}$ be a closed ideal of $\mathcal{A}$. Let $\mathcal{A}$ have UUNP. Does $\mathcal{I}$ have UUNP? (Note that the proof of Theorem 6.1 implies that the answer is affirmative if $\mathcal{I}$ satisfies: $a \in \mathcal{A}, a \mathcal{I}=\{0\}$ implies $a=0$.)

Lemma 2.4. Let $\mathcal{B}$ be a dense subalgebra of $\mathcal{A}$. If $\mathcal{B}$ has UUNP, then $\mathcal{A}$ has UUNP.

Proof. Let $|\cdot|$ be a uniform norm on $\mathcal{A}$. Then $|\cdot| \leq r_{\mathcal{A}}(\cdot) \leq\|\cdot\|$ on $\mathcal{A}$. Let $y \in \mathcal{A}$. Choose a sequence $\left(y_{n}\right)$ in $\mathcal{B}$ such that $\left\|y_{n}-y\right\| \rightarrow 0$. Then $\left|y_{n}-y\right| \rightarrow 0$. Since $\mathcal{B}$ has UUNP, $\left|y_{n}\right|=r_{\mathcal{A}}\left(y_{n}\right)$ for all $n$, and so $|y|=r_{\mathcal{A}}(y)$. Thus $\mathcal{A}$ has UUNP.

Lemma 2.5. Let $\mathcal{I}$ be a spectral synthesis ideal of $U(\mathcal{A})$. If $\mathcal{A} /(\mathcal{A} \cap \mathcal{I})$ has $U U N P$, then $\mathcal{I}=k(h(\mathcal{A} \cap \mathcal{I}))$.

Proof. Let $\mathcal{J}=(\mathcal{A} \cap \mathcal{I})^{-}($closure in $U(\mathcal{A}))$. Then $k(h(\mathcal{J}))=k(h(\mathcal{A} \cap \mathcal{I}))$. Consider the homomorphisms $\phi: \mathcal{A} /(\mathcal{A} \cap \mathcal{I}) \rightarrow U(\mathcal{A}) / \mathcal{I}, \phi(x+(\mathcal{A} \cap \mathcal{I}))=$ $x+\mathcal{I}$, and $\psi: \mathcal{A} /(\mathcal{A} \cap \mathcal{I}) \rightarrow U(\mathcal{A}) / k(h(\mathcal{A} \cap \mathcal{I})), \psi(x+\mathcal{A} \cap \mathcal{I})=x+k(h(\mathcal{A} \cap \mathcal{I}))$. Now $\phi$ is one-one. Also, $\psi$ is one-one. Indeed, since $\mathcal{A} /(\mathcal{A} \cap \mathcal{I})$ has UUNP, $\mathcal{A} \cap \mathcal{I}$ is a spectral synthesis ideal of $\mathcal{A}$, and so $\mathcal{A} \cap \mathcal{I}=k(h(\mathcal{J})) \cap \mathcal{A}$. Thus $\psi(x+\mathcal{A} \cap \mathcal{I})=0$ implies that $x \in k(h(\mathcal{A} \cap \mathcal{I})) \cap \mathcal{A}=k(h(\mathcal{J})) \cap \mathcal{A}=\mathcal{A} \cap \mathcal{I}$, showing that $\psi$ is one-one. Now, as $\mathcal{A} /(\mathcal{A} \cap \mathcal{I})$ has UUNP, the subalgebra $\{x+\mathcal{I}: x \in \mathcal{A}\}$ of $U(\mathcal{A}) / \mathcal{I}$ as well as the subalgebra $\{x+k(h(\mathcal{J})): x \in \mathcal{A}\}$ of $U(\mathcal{A}) / k(h(\mathcal{J}))$ have UUNP. Also, $U(\mathcal{A}) / \mathcal{I}$ is semisimple and $\phi(\mathcal{A} /(\mathcal{A} \cap \mathcal{I}))$ is dense in $U(\mathcal{A}) / \mathcal{I}$. Hence by Lemma $2.4, U(\mathcal{A}) / \mathcal{I}$ also has UUNP. For $x$ in $\mathcal{A}$, $|x+\mathcal{A} \cap \mathcal{I}|_{1}=r_{U(\mathcal{A}) / \mathcal{I}}(x+\mathcal{I})$ and $|x+\mathcal{A} \cap \mathcal{I}|_{2}=r_{U(\mathcal{A}) / k(h(\mathcal{J}))}(x+k(h(\mathcal{J})))$ define uniform seminorms on $\mathcal{A} /(\mathcal{A} \cap \mathcal{I})$. Further, $|\cdot|_{1}$ and $|\cdot|_{2}$ are norms because $\mathcal{I}$ and $k(h(\mathcal{J}))$ are spectral synthesis ideals of $U(\mathcal{A})$. As $\mathcal{A} /(\mathcal{A} \cap \mathcal{I})$ has UUNP, $|\cdot|_{1}=|\cdot|_{2}$ on $\mathcal{A} /(\mathcal{A} \cap \mathcal{I})$.

Suppose, if possible, that $x \in \mathcal{I}$, but $x \notin k(h(\mathcal{J}))$. Choose a sequence $\left(x_{n}\right)$ in $\mathcal{A}$ such that $r_{U(\mathcal{A})}\left(x_{n}-x\right) \rightarrow 0$. Then

$$
\begin{aligned}
0 & =r_{U(\mathcal{A}) / \mathcal{I}}(x+\mathcal{I})=\lim r_{U(\mathcal{A}) / \mathcal{I}}\left(x_{n}+\mathcal{I}\right)=\lim \left|x_{n}+\mathcal{A} \cap \mathcal{I}\right|_{1} \\
& =\lim \left|x_{n}+\mathcal{A} \cap \mathcal{I}\right|_{2}=\lim r_{U(\mathcal{A}) / k(h(\mathcal{J}))}\left(x_{n}+k(h(\mathcal{J}))\right) \\
& =r_{U(\mathcal{A}) / k(h(\mathcal{J}))}(x+k(h(\mathcal{J}))) \neq 0
\end{aligned}
$$


because $U(\mathcal{A}) / k(h(\mathcal{J}))$ is semisimple and $x \notin k(h(\mathcal{J}))$. This contradiction proves that $\mathcal{I}=k(h(\mathcal{J}))=k(h(\mathcal{A} \cap \mathcal{I}))$.

Following [Ba], a *-semisimple Banach *-algebra $\mathcal{B}$ is * ${ }^{*}$-regular if for any closed subset $F$ of the primitive ideal space $\Pi(\mathcal{B})$ of $C^{*}(\mathcal{B})$ and $P \in \Pi(\mathcal{B}) \backslash F$, there exists $x$ in $\mathcal{A}$ such that $\widehat{x}(F)=(0)$ and $\widehat{x}(P) \neq 0$. Here $\widehat{x}(\mathcal{I})=x+\mathcal{I}$ $(\mathcal{I} \in \Pi(\mathcal{B}))$. By $\left[\mathrm{Ba}\right.$, Theorem 2.3 and Proposition 2.4], $\mathcal{B}$ is ${ }^{*}$-regular iff for any $\|\cdot\|_{C^{*}(\mathcal{B})}$-closed ${ }^{*}$-ideal $\mathcal{I}$ of $\mathcal{B}, \mathcal{B} / \mathcal{I}$ has $\mathrm{U} C^{*} \mathrm{NP}$ iff for any closed ideal $\mathcal{I}$ of $C^{*}(\mathcal{B}), \mathcal{I} \cap \mathcal{B}$ is dense in $\mathcal{I}$. The following, which provides a UUNP analogue of this, is one of the main results of this paper.

THEOREM 2.6. The following are equivalent:

(i) $\mathcal{A}$ is regular;

(ii) $U(\mathcal{A})$ is regular, and the quotient algebra $\mathcal{A} / \mathcal{I}$ has $U U N P$ for every spectral synthesis ideal $\mathcal{I}$ of $\mathcal{A}$;

(iii) $U(\mathcal{A})$ is regular, and $\mathcal{I}=k(h(\mathcal{A} \cap \mathcal{I}))$ for every spectral synthesis ideal $\mathcal{I}$ of $U(\mathcal{A})$.

Proof. (i) $\Rightarrow$ (ii). Let $\mathcal{A}$ be regular. Then $U(\mathcal{A})$ is regular. Let $\mathcal{I}$ be a spectral synthesis ideal of $\mathcal{A}$. Then $\mathcal{A} / \mathcal{I}$ is regular and semisimple. As every regular, semisimple, commutative Banach algebra has UUNP, $\mathcal{A} / \mathcal{I}$ has UUNP.

(ii) $\Rightarrow$ (iii). Let $\mathcal{I}$ be a spectral synthesis ideal of $U(\mathcal{A})$. Then $U(\mathcal{A}) / \mathcal{I}$ is semisimple, and so $\mathcal{A} /(\mathcal{A} \cap \mathcal{I})$ is semisimple. By (ii), $\mathcal{A} /(\mathcal{A} \cap \mathcal{I})$ has UUNP. Hence, Lemma 2.5 implies that $\mathcal{I}=k(h(\mathcal{A} \cap \mathcal{I}))$.

(iii) $\Rightarrow$ (i). Let $\Gamma$ be closed subset of $\Delta(\mathcal{A})$ in the Gelfand topology. Since $\Delta(\mathcal{A})$ is topologically homeomorphic to $\Delta(U(\mathcal{A}))$ in the Gelfand topologies, corresponding to the set $\Gamma$, there exists a unique closed subset $\widetilde{\Gamma}$ of $\Delta(U(\mathcal{A}))$ in the Gelfand topology. Since $U(\mathcal{A})$ is regular, $h[k(\widetilde{\Gamma})]=\widetilde{\Gamma}$. To prove that $\mathcal{A}$ is regular, it is enough to show that $h[k(\Gamma)] \subseteq \Gamma$. So let $\varphi \in h[k(\Gamma)]$. Then there exists a unique $\widetilde{\varphi} \in \Delta(U(\mathcal{A}))$ such that $\widetilde{\varphi}=\varphi$ on $\mathcal{A}$. Since $\varphi \in h[k(\Gamma)]$, we have $\widetilde{\varphi}=0$ on $k(\Gamma)$. But $k(\Gamma)=\mathcal{A} \cap k(\widetilde{\Gamma})$. Therefore $\widetilde{\varphi}=0$ on $\mathcal{A} \cap k(\widetilde{\Gamma})$. Since $k(\widetilde{\Gamma})$ is a spectral synthesis ideal, we have $k(\widetilde{\Gamma})=k[h(\mathcal{A} \cap k(\widetilde{\Gamma}))]$ due to the assumption. Hence $\widetilde{\varphi}=0$ on $k(\widetilde{\Gamma})$. Thus $\widetilde{\varphi} \in h[k(\widetilde{\Gamma})]=\widetilde{\Gamma}$ because $U(\mathcal{A})$ is regular. So $\varphi \in \Gamma$. This proves that $\mathcal{A}$ is regular.

A semisimple, commutative Banach algebra $\mathcal{A}$ is an $N$-algebra [Ri, p. 92] if every closed ideal of $\mathcal{A}$ is a spectral synthesis ideal. We omit the proof of the following result.

Proposition 2.7. Consider the following.

(i) $\mathcal{A}$ is regular;

(ii) $U(\mathcal{A})$ is regular, and for every spectral synthesis ideal $\mathcal{I}$ of $U(\mathcal{A})$, $\mathcal{A} \cap \mathcal{I}$ is dense in $\mathcal{I}$. 
Then (ii) implies (i). Further, if $U(\mathcal{A})$ is an $N$-algebra, then (i) implies (ii).

Note that if $\mathcal{A}$ is a hermitian, semisimple, commutative Banach *-algebra, then $U(\mathcal{A})=C_{0}(\Delta(\mathcal{A}))$, which is an $N$-algebra. It would be interesting to find an example of a $u \mathcal{B}$-algebra other than $C_{0}(X)$, which is an $N$-algebra.

By [BhDe1], if $\operatorname{dim} \mathcal{A}>1$ and $\mathcal{A}$ has UUNP, then $\mathcal{A}$ cannot be an integral domain. The following shows that the converse does not hold, thereby answering a query in [BhDe1, p. 581].

Proposition 2.8. There exists a semisimple commutative Banach algebra $\mathcal{A}$ having the following properties:

(1) $\operatorname{dim} \mathcal{A}>1$

(2) $\mathcal{A}$ does not have UUNP;

(3) $\mathcal{A}$ is not an integral domain.

Proof. Let $\left(\mathcal{A}_{1},\|\cdot\|_{1}\right)$ and $\left(\mathcal{A}_{2},\|\cdot\|_{2}\right)$ be unital, semisimple commutative Banach algebras such that $\mathcal{A}_{1}$ does not have UUNP and $\mathcal{A}_{2}$ is not an integral domain. Define $\mathcal{A}=\mathcal{A}_{1} \times \mathcal{A}_{2}$, with co-ordinatewise operations and the norm $\left\|\left(a_{1}, a_{2}\right)\right\|=\max \left(\left\|a_{1}\right\|_{1},\left\|a_{2}\right\|_{2}\right)$. Then $\mathcal{A}$ is not an integral domain. Let $\mathcal{I}=\mathcal{A}_{1} \times\{0\}$. Then $k(h(\mathcal{I}))=\mathcal{I}$, which does not have UUNP. Hence $\mathcal{A}$ cannot have UUNP due to Proposition 2.3.

We recall that for semisimple Banach algebras, regularity implies weak regularity implies UUNP, and that these implications are strict. Weak regularity, introduced in [BhDe1], seems to be of independent interest. By [BhDe1, p. 581], $\mathcal{A}$ is weakly regular iff $\partial(\mathcal{A})=\Delta(\mathcal{A})$ and $\mathcal{A}$ has UUNP. The following gives a large class of weakly regular, non-regular Banach algebras.

Proposition 2.9. Let $\left(\mathcal{B},\|\cdot\|_{\mathcal{B}}\right)$ be a unital semisimple commutative Banach algebra. Let $\Omega=\Delta(\mathcal{B}) \times[0,1]$. Let $\mathcal{A}=\{f \in C(\Omega): f(\varphi, 0)=\widehat{b}(\varphi)$ $(\varphi \in \Delta(\mathcal{B}))$ for some $b \in \mathcal{B}\}$ with norm $\|f\|=\max \left(\|f\|_{\Omega},\|f(\cdot, 0)\|_{\mathcal{B}}\right)$, where $\|\cdot\|_{\Omega}$ denotes the supnorm on $\Omega$. Then:

(1) $(\mathcal{A},\|\cdot\|)$ is a semisimple commutative Banach algebra with pointwise operations.

(2) $\Delta(\mathcal{A}) \cong \Omega$ and $\partial(\mathcal{A})=\Delta(\mathcal{A})$.

(3) $\mathcal{A}$ has $S E P$, and hence UUNP.

(4) $\mathcal{A}$ is weakly regular.

(5) $\mathcal{A}$ is a uB-algebra iff $\mathcal{B}$ is a uB-algebra iff $\|\cdot\|=\|\cdot\|_{\Omega}$ on $\mathcal{A}$.

(6) $\mathcal{A}$ is regular iff $\mathcal{B}$ is regular.

(7) $\mathcal{A}$ is hermitian with complex conjugation iff $\mathcal{B}$ is hermitian with respect to some involution. 
Proof. (1) This is clear.

$(2)$ Define $T: \Omega \rightarrow \Delta(\mathcal{A})$ as $T(x, t)=\varphi_{x, t}$ where $\varphi_{x, t}(f)=f(x, t)$ $(f \in \mathcal{A})$. Clearly $T$ is continuous. To show that $T$ is one-one, let $\left(x_{1}, t_{1}\right) \neq$ $\left(x_{2}, t_{2}\right)$. If $t_{1}=t_{2}=0$, then $x_{1} \neq x_{2}$. Since $\mathcal{B}$ is semisimple, there exists $b \in \mathcal{B}$ such that $\widehat{b}\left(x_{1}\right) \neq \widehat{b}\left(x_{2}\right)$. But $b$ has an extension to $\Omega$ belonging to $\mathcal{A}$. Now, if one of $t_{1}, t_{2}$ is non-zero, say $t_{1} \neq 0$, then there exists a continuous function $f: \Omega \rightarrow[0,1]$ such that $f\left(x_{1}, t_{1}\right)=1, f(K)=\{0\}$, where $K=\Delta(\mathcal{B}) \times\{0\} \cup\left\{\left(x_{2}, t_{2}\right)\right\}$. Thus $T$ is one-one. Now it is enough to prove that any $\varphi \in \Delta(\mathcal{A})$ is of the form $\varphi=\varphi_{x, t}$ for some $(x, t) \in \Delta(\mathcal{A})$. Define $\mathcal{I}=\{f \in \mathcal{A}: f(\Delta(\mathcal{B}) \times\{0\})=\{0\}\}, \mathcal{J}=\{f \in \mathcal{A}: f(x, t)=f(x, 0)$, $0 \leq t \leq 1\}$. Then $\mathcal{I}$ is a closed ideal of $\mathcal{A}$, and $\mathcal{J}$ is a closed subalgebra of $\mathcal{A}$. In fact, $\mathcal{I} \cong C_{0}(\Delta(\mathcal{B}) \times(0,1])$ and $\mathcal{J} \cong \mathcal{B}$. Let $f \in \mathcal{A}$ and $b \in \mathcal{B}$ be such that $f=\widehat{b}$ on $\Delta(\mathcal{B}) \times\{0\}$. Then $f=(f-\widehat{b})+\widehat{b} \in \mathcal{I}+\mathcal{J}$. Hence $\mathcal{A}=\mathcal{I} \oplus \mathcal{J}$. Since $\mathcal{J}$ contains the identity, $\varphi$ is non-zero on $\mathcal{J}$. So there exists $x \in \Delta(\mathcal{B})$ such that

$$
\varphi(f)=f(x, 0) \quad(f \in \mathcal{J}) .
$$

Now if $\varphi(\mathcal{I})=\{0\}$, then clearly $\varphi=\varphi_{x, 0}$. If $\varphi(\mathcal{I}) \neq\{0\}$, then there exists $(y, t) \in \Delta(\mathcal{B}) \times(0,1]$ such that

$$
\varphi(f)=f(y, t) \quad(f \in \mathcal{I}) .
$$

For $f \in \mathcal{I}$ and $g \in \mathcal{J}$, we have $f(y, t) g(y, t)=(f g)(y, t)=\varphi(f g)=$ $\varphi(f) \varphi(g)=f(y, t) g(x, 0)$. Choosing an $f$ such that $f(y, t) \neq 0$, we have

$$
g(y, t)=g(x, 0) \quad(g \in \mathcal{J}) .
$$

Now let $f=f_{1}+f_{2} \in \mathcal{I} \oplus \mathcal{J}=\mathcal{A}$. Using (A)-(C), we get $\varphi(f)=\varphi\left(f_{1}\right)+$ $\varphi\left(f_{2}\right)=f_{1}(y, t)+f_{2}(x, 0)=f_{1}(y, t)+f_{2}(y, t)=f(y, t)$. Thus $\varphi=\varphi_{y, t}$. This proves that $\Delta(\mathcal{A})=\Omega$. Now $\partial(\mathcal{A})=\Delta(\mathcal{A})$ is obvious.

(3) Let $G=\Delta(\mathcal{B}) \times(0,1]$. Then $\bar{G}=\Delta(\mathcal{A})=\partial(\mathcal{A})$. Let $U \subset G$ be an open subset of $G$, and hence of $\Delta(\mathcal{A})$. Let $(x, t) \in U$. Then there exists a continuous function $f: \Omega \rightarrow[0,1]$ such that $f \equiv 1$ on $\bar{V} \subset U$, but $f \equiv 0$ outside $U$, where $V$ is a compact neighbourhood of $(x, t)$. Thus $f \in \mathcal{A}$, $f(V)=\{1\}$ and $f\left(U^{\mathrm{c}}\right)=\{0\}$. Hence by [Me2, Theorem 7, p. 73], $\mathcal{A}$ has SEP; and hence it has UUNP.

(4) Since $\mathcal{A}$ has UUNP and $\partial(\mathcal{A})=\Delta(\mathcal{A})$, [BhDe1, Theorem 2.3] implies that $\mathcal{A}$ is weakly regular.

(5) This follows from the definition of $\|\cdot\|$ and $\mathcal{A}$ having UUNP.

(6) Suppose $\mathcal{B}$ is not regular. Then there exists a closed set $F \subset \Delta(\mathcal{B})$ and $\varphi \in \Delta(\mathcal{B}) \backslash F$ such that there is no $b \in \mathcal{B}$ satisfying $\widehat{b}(F)=\{0\}, \widehat{b}(\varphi)=1$. Take $F_{1}=F \times\{0\}$ and $(x, t)=(\varphi, 0)$. Then there exists no $f \in \mathcal{A}$ such that $f\left(F_{1}\right)=\{0\}$ and $f(x, t)=1$. Hence $\mathcal{A}$ is not regular. 
Conversely, suppose that $\mathcal{B}$ is regular. Let $F$ be a closed set in $\Delta(\mathcal{A})$ and $(x, t) \in \Delta(\mathcal{A}) \backslash F$. If $t \neq 0$, then $F_{1}=F \cap H$ is closed in $H$ and $(x, t) \in H \backslash F_{1}$, where $H=\Delta(\mathcal{B}) \times(0,1]$. Hence there exists $f \in \mathcal{I}\left(\cong C_{0}(H)\right)$ such that $f(x, t)=1, f\left(F_{1}\right)=(0)$, and hence $f(F)=(0)$. Now suppose that $t=0$. Then there exists an open neighbourhood $U_{x}$ of $x$ in $\Delta(\mathcal{B})$ such that $\bar{U}_{x} \times[0, \varepsilon] \cap F=\emptyset$ for some $\varepsilon>0$. Now define $F_{1}=\Delta(\mathcal{B}) \backslash U_{x}$. Then $x \notin F_{1}$. Since $\mathcal{B}$ is regular, there exists $b \in \mathcal{B}$ such that $\widehat{b}(x)=1$ and $\widehat{b}\left(F_{1}\right)=(0)$. Define $f_{1}(y, t)=\widehat{b}(y)((y, t) \in \Omega)$. Then $f_{1} \in \mathcal{A}$, and $f_{1}((x, 0))=1, f_{1}(F)=(0)$. Let $F_{2}=\Delta(\mathcal{B}) \times[\varepsilon / 2,1]$. Then there exists $f \in C(\Omega)$ such that $f\left(F_{2}\right)=(0)$ and $f(\Delta(\mathcal{B}) \times(0))=1$. Thus $f \in \mathcal{A}$. Take $g=f f_{1} \in \mathcal{A}$. Then $g(x, 0)=1$. For any $(y, s) \in F$, there are two cases. If $0 \leq s<\varepsilon / 2$, then $y \notin U_{x}$, hence $f_{1}(y, s)=\widehat{b}(y)=0$. If $s \geq \varepsilon / 2$, then $(y, s) \in F_{2}$. All these imply that $g(F)=(0)$. Thus $\mathcal{A}$ is regular.

(7) Let $\mathcal{B}$ be hermitian with some involution $b \mapsto b^{*}$. Let $f \in \mathcal{A}$, $f(\varphi, 0)=\widehat{b}(\varphi)$. Then $\overline{f(\varphi, 0)}=\widehat{b^{*}}(\varphi)$. Thus $\mathcal{A}$ is hermitian. Conversely, if $\mathcal{A}$ is hermitian, then $\widehat{b^{*}}(\varphi)=\overline{f(\varphi, 0)}$, for given $f \in \mathcal{A}$, defines a hermitian involution on $\mathcal{B}$.

We call $\mathcal{A} U$-regular if, given a closed set $F \subset \Delta(\mathcal{A})$ and $\varphi \in \partial(\mathcal{A}) \backslash F$, there exists $x \in \mathcal{A}$ such that $\widehat{x}(F)=\{0\}$ and $\widehat{x}(\varphi) \neq 0$. Thus $\mathcal{A}$ is regular iff $\partial(\mathcal{A})=\Delta(\mathcal{A})$ and $\mathcal{A}$ is $U$-regular. By [BhDe1, Theorem 2.3], UUNP is equivalent to weak- $U$-regularity; i.e., given any closed set $F \subset \Delta(\mathcal{A})$ having $\partial(\mathcal{A}) \cap(\Delta(\mathcal{A}) \backslash F) \neq \emptyset$, there exists $x \neq 0$ in $\mathcal{A}$ such that $\widehat{x}(F)=\{0\}$.

In the case of involutive algebras, the following describes the interrelations among UUNP, $\mathrm{UC}^{*} \mathrm{NP}$, weak regularity and hermiticity. We omit its simple proof.

Proposition 2.10. Let $\mathcal{A}$ be a commutative Banach ${ }^{*}$-algebra.

(1) Any two of the following imply the third:

(i) $\mathcal{A}$ has UUNP;

(ii) $\mathcal{A}$ has $U C^{*} N P$;

(iii) $\mathcal{A}$ is hermitian.

(2) Let $\mathcal{A}$ be ${ }^{*}$-semisimple. Then:

(i) If $\mathcal{A}$ has $U U N P$, then $\mathcal{A}$ has $U C^{*} N P$ and $\mathcal{A}$ is hermitian;

(ii) $\mathcal{A}$ is weakly regular iff $\mathcal{A}$ is hermitian and has UUNP.

(3) Let $\mathcal{A}$ be hermitian. Then $A$ is $U$-regular iff $\mathcal{A}$ is ${ }^{*}{ }^{*}$-regular iff $\mathcal{A}$ is regular.

By [BD, Theorem 35.3, p. 188] and the above, it follows that a ${ }^{*}$-semisimple, non-hermitian commutative Banach *-algebra admits more than one uniform norms. 
Proposition 2.11. (1) Suppose that there exists a topological group $G$ acting on $\Delta(\mathcal{A})$ from the left satisfying the following conditions.

(A) For each $x \in \mathcal{A}, g \in G$, there exists $y \in \mathcal{A}$ such that $\widehat{y}(\varphi)=\widehat{x}(g \cdot \varphi)$ for all $\varphi$ in $\Delta(\mathcal{A})$.

(B) For each $\varphi \in \Delta(\mathcal{A}),\{U \cdot \varphi: U$ is a neighbourhood of identity in $G\}$ form an open neighbourhood base for $\varphi$ in $\Delta(\mathcal{A})$.

Then $\mathcal{A}$ has UUNP iff $\mathcal{A}$ is $U$-regular.

(2) Further, assume that $\partial(\mathcal{A})=\Delta(\mathcal{A})$. Then $\mathcal{A}$ has UUNP iff $\mathcal{A}$ is regular.

Proof. (1) Assume that $\mathcal{A}$ has UUNP. Then $\mathcal{A}$ is weakly $U$-regular. Let $F \subset \Delta(\mathcal{A})$ be a closed set, and $\varphi \in \partial(\mathcal{A}) \backslash F$. Set $V=\Delta(\mathcal{A}) \backslash F$. By $(\mathrm{B})$, there exists an open neighbourhood $U$ of $e$ in $G$ such that $U \cdot \varphi \subset V$. By standard topological group theory, there exists an open neighbourhood $W$ of $e$ in $G$ such that $W^{2} \subset U$ and $W^{-1}=W$. Now $\Delta(\mathcal{A}) \backslash W \varphi$ is a closed set in $\Delta(\mathcal{A})$ such that $\varphi \in \partial(\mathcal{A}) \cap(W \varphi) \neq \emptyset$. By the weak $U$-regularity of $\mathcal{A}$, there exist $x \neq 0$ in $\mathcal{A}$ and $g \in W$ such that $\widehat{x}(\Delta(\mathcal{A}) \backslash W \varphi)=0$ and $\widehat{x}(g \cdot \varphi) \neq 0$. Note that, if $\psi \in F$, then $g \cdot \psi \notin W \varphi$ (since $g \cdot \psi \in W \varphi$ implies that $\left.\psi \in g^{-1} W \varphi \subset W^{2} \varphi \subset V\right)$. Define $\widehat{x}_{g}(\eta):=\widehat{x}(g \cdot \eta)(\eta \in \Delta(\mathcal{A}))$. This defines an element $y \in \mathcal{A}$ such that $\widehat{y}=\widehat{x}_{g}$. Then $\widehat{y}(\varphi)=\widehat{x}(g \cdot \varphi) \neq 0$, and $\widehat{y}(\psi)=\widehat{x}(g \psi) \in \widehat{x}\left((W \varphi)^{\mathrm{c}}\right)=(0)$ for all $\psi \in F$. Thus $\mathcal{A}$ is $U$-regular. This proves (1).

(2) This follows from (1).

The following supplements the fact that every Segal algebra on a locally compact abelian group has UUNP [BhDe1].

Corollary 2.12. Let $G$ be a locally compact abelian group. Suppose $\mathcal{A}$ is a dense subalgebra of $L^{1}(G)$ satisfying the following:

(1) $\mathcal{A}$ is a Banach algebra with respect to some norm;

(2) $\Delta(\mathcal{A})=\widehat{G}$ (the dual group of $G)$;

(3) $x \in \mathcal{A}, \varphi \in \widehat{G}$ implies $\bar{\varphi} \cdot x \in \mathcal{A}$, where $(\bar{\varphi} \cdot x)(g)=\overline{\varphi(g)} x(g)$;

(4) $\partial(\mathcal{A})=\Delta(\mathcal{A})$.

Then $\mathcal{A}$ is regular iff $\mathcal{A}$ has UUNP.

Proposition 2.13. Let $\Delta(\mathcal{A})$ be homeomorphic to either a subset of $\mathbb{R}$ or a subset of the unit circle. Then $\mathcal{A}$ is regular iff $\mathcal{A}$ is weakly regular.

Proof. Suppose that $\Delta(\mathcal{A}) \subset \mathbb{R}$. Let $\mathcal{A}$ be weakly regular, so that $\mathcal{A}$ has UUNP. We show that $\mathcal{A}$ is regular. Let $\mathcal{A}_{e}$ be the algebra obtained by adjoining an identity to $\mathcal{A}$. By [BhDe2], $\mathcal{A}_{e}$ has UUNP, $\partial\left(\mathcal{A}_{e}\right)=\Delta\left(\mathcal{A}_{e}\right)$, and $\mathcal{A}_{e}$ is weakly regular. Thus we can assume that $\mathcal{A}$ has an identity. Then 
$\Delta(\mathcal{A})$ is a compact subset of $\mathbb{R}$. For any $\alpha \in \mathbb{R}$, let $K_{\alpha}=\Delta(\mathcal{A}) \cap(-\infty, \alpha]$, $J_{\alpha}=\Delta(\mathcal{A}) \cap[\alpha, \infty)$. Fix $\alpha, \beta$ in $\Delta(\mathcal{A})$ such that $\alpha<\beta$.

CASE (i): There exists $\delta \notin \Delta(\mathcal{A})$ such that $\alpha<\delta<\beta$. Then $J_{\delta}$ is open and compact in $\Delta(\mathcal{A})$. Hence by Shilov's idempotent theorem, there exists an idempotent $e \in \mathcal{A}$ such that $\widehat{e}\left(K_{\delta}\right)=(0)$ and $\widehat{e}\left(J_{\delta}\right)=(1)$.

CASE (ii): $(\alpha, \beta) \subset \Delta(\mathcal{A})$. Let $\Gamma=\{\varphi \in \Delta(\mathcal{A}): \widehat{x}(\varphi)=0$ for all $x \in \mathcal{A}$ such that $\left.\widehat{x}\left(K_{\alpha}\right)=0\right\}=h\left(k\left(K_{\alpha}\right)\right)$. Suppose that $\beta \in \Gamma$. Since $\mathcal{A}$ is weakly regular, there exists $y \in \mathcal{A}$ such that $\widehat{y}(\varphi)=0(\varphi \in \Delta(\mathcal{A}) \backslash(\alpha, \beta))$ and $y \neq 0$. Hence there exists $\tau \notin \Gamma, \alpha<\tau<\beta$, such that $\widehat{y}(\tau) \neq 0$. Let $\mathcal{I}=\{x \in \mathcal{A}: \widehat{x}(\Gamma)=0\}=k(h(k(\Gamma)))=k(\Gamma)$, which is a semisimple ideal of $\mathcal{A}$, and $\Delta(\mathcal{A} / \mathcal{I}) \cong \Gamma$. As $\widehat{y}(\tau) \neq 0$ and $\Delta(\mathcal{A} / \mathcal{I}) \cong \Gamma$, Case (i) applies to $\mathcal{A} / \mathcal{I}$ showing that there exists an idempotent $h+\mathcal{I} \in \mathcal{A} / \mathcal{I}$ such that $(h+\mathcal{I})^{\wedge}(\varphi)=0(\varphi \in \Gamma, \varphi<\tau),(h+\mathcal{I})^{\wedge}(\varphi)=1(\varphi \in \Gamma, \tau<\varphi)$. But then $\widehat{h}\left(K_{\alpha}\right)=\{0\}$ and $\widehat{h}(\beta)=1$. Thus $\mathcal{A}$ is regular.

REMARK 2.14. Albrecht $[\mathrm{A}]$ has shown that every commutative Banach algebra $\mathcal{A}$ contains a largest closed subalgebra $\operatorname{Reg} \mathcal{A}$ which is regular. If $\mathcal{A}$ is semisimple, then $\operatorname{Reg} \mathcal{A}$ has UUNP. For $a \in \mathcal{A}$, let $L_{a}: \mathcal{A} \rightarrow \mathcal{A}$ be the multiplication operator $L_{a} x=a x$. Let $\operatorname{Dec} \mathcal{A}=\left\{a \in \mathcal{A}: L_{a}\right.$ is a decomposable operator $\}$. By [LN], Dec $\mathcal{A}$ is a closed subalgebra of $\mathcal{A}$ and $\operatorname{Reg} \mathcal{A} \subset \operatorname{Dec} \mathcal{A}$. Does the Banach algebra $\operatorname{Dec} \mathcal{A}$ have UUNP or is weakly regular? This is a weaker version of the open problem whether $\operatorname{Reg} \mathcal{A}=$ $\operatorname{Dec} \mathcal{A}$ [LN, p. 203]. Does $\mathcal{A}$ contain a largest closed subalgebra $U_{\mathrm{np}}(\mathcal{A})$ which has UUNP? The following example shows that, if it exists, it can be much larger than Dec $\mathcal{A}$. Let $0<r<1$ and $\mathcal{A}=\left\{f \in C\left(\bar{\Delta}_{1}\right): f\right.$ is holomorphic in $\left.\Delta_{r}\right\}$. Then $\mathcal{A}$ has UUNP and $\operatorname{Reg} \mathcal{A}=\operatorname{Dec} \mathcal{A} \neq \mathcal{A}$. Indeed, let $\mathcal{I}=\{f \in \mathcal{A}$ : $f=0$ on $\left.\Delta_{r}\right\}$. Then $\mathcal{I}=C_{0}(H)$, where $H=\{z \in \mathbb{C}: r<|z| \leq 1\}$, so that $\mathcal{I}$ is regular. Hence $\mathcal{I}_{e}$, obtained by adjoining an identity to $\mathcal{I}$, is regular. Thus $\mathcal{I}_{e} \subseteq \operatorname{Reg} \mathcal{A} \subseteq \operatorname{Dec} \mathcal{A}$. By [N2, Theorem 1.2], $\operatorname{Dec} \mathcal{A}=\{a \in \mathcal{A}: \widehat{a}$ is hull-kernel continuous on $\Delta(\mathcal{A})\}$. Now if $f \notin \mathcal{I}_{e}$, then $f$ is not constant on $\Delta_{r}$. Let $z \in \Delta_{r}, \bar{U}=\overline{U(z, \varepsilon)} \subset \Delta_{r}, F=f(\bar{U})$. Then $F$ is a Gelfand closed subset of $\Delta(\mathcal{A})=\bar{\Delta}_{1}$. But $h\left(k\left(f^{-1}(F)\right)\right)=\bar{\Delta}_{r} \neq f^{-1}(F)$ showing that $f^{-1}(F)$ is not hull-kernel closed. Thus $\operatorname{Dec} \mathcal{A} \subseteq \mathcal{I}_{e}$. Hence $\operatorname{Dec} \mathcal{A}=\mathcal{I}_{e}$, while $U_{\text {np }}(\mathcal{A})=\mathcal{A}$.

3. Tensor products. Let $\mathcal{A}$ and $\mathcal{B}$ be ${ }^{*}$-semisimple Banach ${ }^{*}$-algebras. It follows from [HKV, Theorems 3.3 and 3.4] that the algebraic tensor product $\mathcal{A} \otimes \mathcal{B}$ has $\mathrm{U} C^{*} \mathrm{NP}$ (respectively, is ${ }^{*}$-regular) iff each of $\mathcal{A}, \mathcal{B}$ and $C^{*}(\mathcal{A}) \otimes C^{*}(\mathcal{B})$ has $\mathrm{U} C^{*} \mathrm{NP}$ (respectively, is ${ }^{*}$-regular). Taking $\mathcal{A}$ to be a non-nuclear $C^{*}$-algebra, there exists a $C^{*}$-algebra $\mathcal{B}$ such that $\mathcal{A} \otimes \mathcal{B}$ fails to have $\mathrm{U} C^{*} \mathrm{NP}$, even though $\mathcal{A}$ and $\mathcal{B}$ obviously have $\mathrm{U} C^{*} \mathrm{NP}$. The following shows that UUNP behaves canonically with respect to tensor products; 
this should also be compared with the result that, if $\alpha$ is a submultiplicative cross-norm on $\mathcal{A} \otimes \mathcal{B}$ dominating the injective cross-norm $\lambda$, then the completion $\mathcal{A} \widehat{\otimes}_{\alpha} \mathcal{B}$ is regular precisely when $\mathcal{A}$ and $\mathcal{B}$ are regular [KN, p. 226].

Theorem 3.1. Let $\mathcal{A}$ and $\mathcal{B}$ be semisimple commutative Banach algebras. Then $\mathcal{A} \otimes \mathcal{B}$ has UUNP iff each of $\mathcal{A}$ and $\mathcal{B}$ has UUNP.

Proof. Suppose that $\mathcal{A}$ and $\mathcal{B}$ have UUNP. For $z \in \mathcal{A} \otimes \mathcal{B}$, define

$$
|z|_{\infty}=\sup \{|\varphi(z)|: \varphi \in \Delta(\mathcal{A} \otimes \mathcal{B})\} .
$$

Since each $\varphi \in \Delta(\mathcal{A} \otimes \mathcal{B}) \cong \Delta(\mathcal{A}) \times \Delta(\mathcal{B})$ is continuous with respect to the projective tensor product $p(\cdot)$ on $\mathcal{A} \otimes \mathcal{B}$, we have $|z|_{\infty} \leq p(z)$ for all $z \in \mathcal{A} \otimes \mathcal{B}$. Hence, as in the proof of [BD, Proposition 20, p. 237], $|\cdot|_{\infty}$ is a uniform norm on $\mathcal{A} \otimes \mathcal{B}$. Let $|\cdot|$ be any uniform norm on $\mathcal{A} \otimes \mathcal{B}$. Then there exists a closed subset $F$ of $\Delta(\mathcal{A} \otimes \mathcal{B})$ such that

$$
|z|=\sup \{|\varphi(z)|: \varphi \in F\} \quad(z \in \mathcal{A} \otimes \mathcal{B}) ;
$$

in fact, $F=\{\varphi \in \Delta(\mathcal{A} \otimes \mathcal{B}): \varphi$ is $|\cdot|$-continuous $\}$. So it is clear that $|\cdot| \leq|\cdot|_{\infty}$ on $\mathcal{A} \otimes \mathcal{B}$. Next we prove that $|\cdot|_{\infty} \leq|\cdot|$. For this, it is enough to prove that $\partial(\mathcal{A} \otimes \mathcal{B}) \subseteq F$. Suppose, if possible, $F$ does not contain $\partial(\mathcal{A} \otimes \mathcal{B})$. By $[\mathrm{BD}$, Theorem 19, p. 236], $\partial(\mathcal{A} \otimes \mathcal{B}) \cong \partial(\mathcal{A}) \times \partial(\mathcal{B})$. So there exist $\varphi \in \partial(\mathcal{A})$ and $\psi \in \partial(\mathcal{B})$ such that $\varphi \otimes \psi \in \partial(\mathcal{A} \otimes \mathcal{B})$, but $\varphi \otimes \psi \notin F$. This, in turn, implies that there exist open sets $U$ and $V$ in $\Delta(\mathcal{A})$ and $\Delta(\mathcal{B})$ respectively such that $(U \otimes V) \cap F=\emptyset$, where $U \otimes V=\{\gamma \otimes \delta: \gamma \in U$ and $\delta \in V\}$. Set $F_{\mathcal{A}}=\Delta(\mathcal{A}) \backslash U$ and $F_{\mathcal{B}}=\Delta(\mathcal{B}) \backslash V$. Then $F_{\mathcal{A}}$ and $F_{\mathcal{B}}$ do not contain $\partial(\mathcal{A})$ and $\partial(\mathcal{B})$, respectively. Since $\mathcal{A}$ and $\mathcal{B}$ are assumed to have UUNP, there exist non-zero $a \in \mathcal{A}$ and non-zero $b \in \mathcal{B}$ such that $\widehat{a}=0$ on $F_{\mathcal{A}}$ and $\widehat{b}=0$ on $F_{\mathcal{B}}$. Then $a \otimes b \neq 0$. On the other hand, for any $\theta \in F, \theta(a \otimes b)=0$, and so $|a \otimes b|=0$, which implies that $a \otimes b=0$ because $|\cdot|$ is a norm. This is a contradiction. Hence $\partial(\mathcal{A} \otimes \mathcal{B}) \subset F$, i.e., $|\cdot|_{\infty} \leq|\cdot|$ and so $\mathcal{A} \otimes \mathcal{B}$ has UUNP.

Conversely, suppose that $\mathcal{A} \otimes \mathcal{B}$ has UUNP. Let $F \subseteq \Delta(\mathcal{A})$ be a closed set of uniqueness for $\mathcal{A}$. Then $F \otimes \partial(\mathcal{B})=\{\varphi \otimes \psi: \varphi \in F, \psi \in \partial(\mathcal{B})\} \subseteq \Delta(\mathcal{A} \otimes \mathcal{B})$ is a closed set of uniqueness for $\mathcal{A} \otimes \mathcal{B}$. Since $\mathcal{A} \otimes \mathcal{B}$ has UUNP, we have $\partial(\mathcal{A}) \times \partial(\mathcal{B}) \cong \partial(\mathcal{A} \otimes \mathcal{B}) \subseteq F \otimes \partial(\mathcal{B})$, which implies that $\partial(\mathcal{A}) \subseteq F$. Thus $\partial(\mathcal{A})$ is the smallest closed set of uniqueness for $\mathcal{A}$, and so $\mathcal{A}$ has UUNP by [BhDe1, Theorem 2.3]. Similarly, $\mathcal{B}$ has UUNP.

Corollary 3.2. Let $\alpha$ be a submultiplicative norm on $\mathcal{A} \otimes \mathcal{B}$ such that $\mathcal{A} \widehat{\otimes}_{\alpha} \mathcal{B}$ is semisimple. If each of $\mathcal{A}$ and $\mathcal{B}$ has UUNP, then $\mathcal{A} \widehat{\otimes}_{\alpha} \mathcal{B}$ has UUNP.

Proof. Let $K=\mathcal{A} \widehat{\otimes}_{\alpha} \mathcal{B}, W=\mathcal{A} \otimes \mathcal{B}$. Let $|\cdot|$ be a uniform norm on $K$. Then $|\cdot| \leq r_{K}(\cdot) \leq \alpha(\cdot)$ on $K$. By the above theorem, $|\cdot|_{\infty}=|\cdot|=r_{K}(\cdot)$ on $W$, and hence $|\cdot|=r_{K}(\cdot)$ on $K$. The corollary follows. 
It should be noted that, if $\mathcal{A}$ and $\mathcal{B}$ are semisimple, then $\mathcal{A} \widehat{\otimes}_{\alpha} \mathcal{B}$ may fail to be semisimple, though $\mathcal{A} \otimes \mathcal{B}$ is semisimple [BD, p. 237]. In the above, if $\mathcal{A} \widehat{\otimes}_{\alpha} \mathcal{B}$ has UUNP, do both $\mathcal{A}$ and $\mathcal{B}$ have UUNP? If $\mathcal{A}$ and $\mathcal{B}$ have UUNP, is $\mathcal{A} \widehat{\otimes}_{\alpha} \mathcal{B}$ necessarily semisimple?

REMARK 3.3. The above shows that $C^{*}$-norms and uniform norms do not behave identically in tensor products. Here is another instance. Let $\mathcal{A}$ and $\mathcal{B}$ be $C^{*}$-algebras. It is well known that every $C^{*}$-norm on the algebraic tensor product $\mathcal{A} \otimes \mathcal{B}$ is a cross norm. However, a uniform norm on the tensor product of $u B$-algebras need not be a cross norm. Take $\mathcal{A}=\mathcal{B}=\mathcal{A}(\bar{\Delta})$, the disc algebra. Let $F=\{(z, w):|z|=|w|=1 / 2\} \subset \bar{\Delta} \times \bar{\Delta}=\Delta(\mathcal{A} \otimes \mathcal{A})$. Then for $h=\sum_{i=1}^{n} f_{i} \otimes g_{i} \in \mathcal{A} \otimes \mathcal{B}$, the formula $|h|_{F}=\sup \left\{\left|\sum f_{i}(z) g_{i}(w)\right|\right.$ : $(z, w) \in F\}$ defines a uniform norm on $\mathcal{A} \otimes \mathcal{B}$. For any non-constant $f, g$ in $\mathcal{A}(\bar{\Delta})$, we have $|f \otimes g|_{F}=\sup \{|f(z)|:|z|=1 / 2\} \sup \{|g(z)|:|z|=1 / 2\}<$ $\|f\|\|g\|,\|\cdot\|$ denoting the supnorm on $\mathcal{A}(\bar{\Delta})$. On the other hand, let $\mathcal{A}$ and $\mathcal{B}$ be $u B$-algebras with UUNP. Let $|\cdot|$ be any uniform norm on $\mathcal{A} \otimes \mathcal{B}$. Then, for each $x \in \mathcal{A}$ and $y \in \mathcal{B},|x \otimes y|=\sup \left\{\left|\varphi_{1}(x)\right| \cdot\left|\varphi_{2}(y)\right|: \varphi_{1} \in \Delta(\mathcal{A})\right.$, $\left.\varphi_{2} \in \Delta(\mathcal{B})\right\}=r_{\mathcal{A}}(x) r_{\mathcal{B}}(y)=|x| \cdot|y|$.

The above theorem applies to a number of vector-valued Banach function algebras. Let $\mathcal{A}$ be a semisimple commutative Banach algebra. Let $X$ be a locally compact Hausdorff space. Then the Banach algebra $C_{0}(X, \mathcal{A})$ of $\mathcal{A}$ valued continuous functions vanishing at infinity is $C_{0}(X, \mathcal{A})=C_{0}(X) \widehat{\otimes}_{\lambda} \mathcal{A}$, which has UUNP provided $\mathcal{A}$ has UUNP. Let $G$ be a locally compact abelian group. The convolution algebra $L^{1}(G, \mathcal{A})=L^{1}(G) \widehat{\otimes}_{\gamma} \mathcal{A}$ has UUNP if $\mathcal{A}$ has UUNP. Further, let $G$ be compact and $1<p<\infty$. Let $L^{p}(G, \mathcal{A})$ be the convolution Banach algebra of measurable functions $f: G \rightarrow \mathcal{A}$ with $|f|=\left[\int_{G}\|f(s)\|^{p} d \mu\right]^{1 / p}<\infty$. Then $L^{p}(G, \mathcal{A})$ is known to be realizable as a completed tensor product $L^{p}(G) \widehat{\otimes}_{\eta} \mathcal{A}$, where, for $f=\sum x_{i} \otimes y_{i}$, the norm $\eta_{p}(f)=\left[\int\left\|\sum x_{i}(g) y_{i}\right\|^{p} d \mu\right]^{1 / p}$. Now $L^{p}(G)$ is a Segal algebra on $G$, and hence has UUNP [BhDe1]. Thus $L^{p}(G, \mathcal{A})$ has UUNP if $\mathcal{A}$ has UUNP. Let $[a, b]$ be a compact interval in $\mathbb{R}$. Let $C^{n}([a, b], \mathcal{A})$ be the Banach algebra of $n$-times continuously differentiable $\mathcal{A}$-valued functions $f:[a, b] \rightarrow \mathcal{A}$ having norm $\|f\|=\sum_{k=0}^{n}(1 / k !)\left\|f^{(k)}\right\|_{\infty}$. Let $0<\alpha<1$. Let $\operatorname{Lip}_{\alpha}([a, b], \mathcal{A})$ consist of all $\mathcal{A}$-valued functions $f:[a, b] \rightarrow \mathcal{A}$ such that $s_{\alpha}(f)<\infty$, where $s_{\alpha}(f)=$ $\sup \left\{\|f(u)-f(v)\| /|u-v|^{\alpha}: u \neq v\right.$ in $\left.[a, b]\right\}$. The vector-valued Lipschitz algebra $\operatorname{Lip}_{\alpha}([a, b], \mathcal{A})$ is a Banach algebra with norm $\|f\|_{\alpha}=\|f\|_{\infty}+s_{\alpha}(f)$. Let $\operatorname{lip}_{\alpha}([a, b], \mathcal{A})=\left\{f \in \operatorname{Lip}_{\alpha}([a, b], \mathcal{A}): m_{\alpha}(f, \delta) \rightarrow 0\right.$ as $\left.\delta \rightarrow 0\right\}$ where $m_{\alpha}(f, \delta)=\sup \left\{\|f(u)-f(v)\| /|u-v|^{\alpha}: u, v\right.$ in $\left.[a, b], 0<|u-v| \leq \delta\right\}$. As discussed in $[\mathrm{KN}], C^{n}([a, b], \mathcal{A})$ and $\operatorname{lip}_{\alpha}([a, b], \mathcal{A})$ can be represented as completed tensor products with canonical norms, and hence both have UUNP if $\mathcal{A}$ has UUNP. 
4. Beurling algebras. Let $G$ be a locally compact abelian group. A weight on $G$ is a continuous function $\omega: G \rightarrow(0, \infty)$ such that $\omega(s+t)$ $\leq \omega(s) \omega(t)(s, t \in G)$. Then $L^{1}(G, \omega)$ is the set of all equivalence classes of complex-valued, Borel measurable functions on $G$ such that $f \omega \in L^{1}(G)$. Then $L^{1}(G, \omega)$ is a commutative Banach algebra with the convolution product, the norm being the weighted $L^{1}$-norm; namely,

$$
\|f\|=\int_{G}|f(s)| \omega(s) d \lambda(s) \quad\left(f \in L^{1}(G, \omega)\right),
$$

where $\lambda$ is the Haar measure on $G$. The following is the main result of this section.

Theorem 4.1. Let $L^{1}(G, \omega)$ be semisimple. Then $L^{1}(G, \omega)$ is regular iff it has UUNP.

Let $0 \leq \gamma \leq 1, \omega_{\gamma}(s)=\exp \left(|s|^{\gamma}\right)$ on $\mathbb{R}$. It follows from [Do] that $L^{1}\left(\mathbb{R}, \omega_{\gamma}\right), \gamma<1$, has UUNP; whereas $L^{1}\left(\mathbb{R}, \omega_{1}\right)$ fails to have UUNP.

In order to prove the above theorem we shall need some preliminaries. By an $\omega$-bounded generalized character on $G$ we mean a non-zero, complexvalued, continuous function $\alpha$ on $G$ such that

$$
|\alpha(s)| \leq \omega(s) \quad(s \in G) \quad \text { and } \quad \alpha(s+t)=\alpha(s) \alpha(t) \quad(s, t \in G) .
$$

Let $H(G, \omega)$ denote the set of all $\omega$-bounded generalized characters on $G$. We do not know whether $H(G, \omega)$ is always non-empty. However, we have the following.

Lemma 4.2. Let $G$ be an LCA group, and let $\omega$ be a weight on $G$. Then the following are equivalent:

(i) $H(G, \omega)$ is non-empty;

(ii) $L^{1}(G, \omega)$ is semisimple;

(iii) $L^{1}(G, \omega)$ is not a radical Banach algebra.

Proof. (i) $\Rightarrow$ (ii). Suppose that $H(G, \omega)$ is non-empty. Fix $\alpha$ in $H(G, \omega)$. Then, for any $s \in G, \alpha(s) \neq 0$. For $\theta \in \widehat{G}$, define $\alpha_{\theta}: L^{1}(G, \omega) \rightarrow \mathbb{C}$ as $\alpha_{\theta}(f)=\widehat{f}\left(\alpha_{\theta}\right)=\int_{G} f(s) \alpha(s) \theta(s) d \lambda(s)\left(f \in L^{1}(G, \omega)\right)$. Then $\alpha_{\theta}$ is a complex homomorphism on $L^{1}(G, \omega)$. Let $f \in \operatorname{rad} L^{1}(G, \omega)$. Since $f \alpha$ belongs to $L^{1}(G)$ and $(f \alpha)^{\wedge}(\theta)=\widehat{f}\left(\alpha_{\theta}\right)=0$ for all $\theta \in \widehat{G}, f \alpha=0$ a.e. Hence $f=0$ a.e. because $\alpha(s) \neq 0$ for each $s \in G$. Thus $L^{1}(G, \omega)$ is semisimple.

(ii) $\Rightarrow$ (iii). This is obvious.

(iii) $\Rightarrow\left(\right.$ i). Suppose that $L^{1}(G, \omega)$ is not radical. Then there exists a complex homomorphism on $L^{1}(G, \omega)$, say $\varphi$. Since $L^{1}(G, \omega)^{*}=L^{\infty}(G, 1 / \omega)$, there exists $\alpha \in L^{\infty}(G, 1 / \omega)$ such that

$$
\varphi(f)=\int_{G} f(s) \alpha(s) d \lambda(s) \quad\left(f \in L^{1}(G, \omega)\right) .
$$


By a standard argument as for $L^{1}(G), \alpha$ is an $\omega$-bounded generalized character on $G$. Thus $H(G, \omega)$ is non-empty.

COROLlary 4.3. In each of the following cases, $L^{1}(G, \omega)$ is semisimple:

(1) $G=\mathbb{R}$, the real line;

(2) $G$ is discrete;

(3) $\omega(s) \geq 1$ for all $s \in G$.

Proof. (1) Define

$\sigma_{1}=\sup \{-\log \omega(-s) / s: s>0\}, \quad \sigma_{2}=\inf \{\log \omega(s) / s: s>0\}$.

Then $-\infty<\sigma_{1} \leq \sigma_{2}<\infty$. Let $z \in \mathbb{C}$ such that $\sigma_{1} \leq \operatorname{Re} z \leq \sigma_{2}$. Define

$$
\alpha_{z}(f)=\int_{\mathbb{R}} f(s) \exp (-z s) d \lambda(s) \quad\left(f \in L^{1}(\mathbb{R}, \omega)\right) .
$$

Then $\alpha_{z} \in H(\mathbb{R}, \omega)$. Hence $L^{1}(\mathbb{R}, \omega)$ is semisimple.

(2) For $s \in G$, define $\delta_{s}(s)=1$ and $\delta_{s}(t)=0$ for all $t \neq s$. Then $\delta_{s} \in L^{1}(G, \omega)$ and

$$
\lim _{n \rightarrow \infty}\left\|\delta_{s}^{n}\right\|^{1 / n}=\lim _{n \rightarrow \infty}\left\|\delta_{n s}\right\|^{1 / n}=\lim _{n \rightarrow \infty} \omega(n s)^{1 / n} \neq 0 .
$$

This implies that $L^{1}(G, \omega)$ is not a radical Banach algebra. Hence it is semisimple.

(3) In this case $L^{1}(G, \omega) \subseteq L^{1}(G)$. The conclusion follows from the fact that a subalgebra of a semisimple, commutative Banach algebra is semisimple.

The following technical result (and its proof) are analogous to the corresponding result for $L^{1}(G)$ [La2, Theorem 4.7.5, p. 123].

Proposition 4.4. The Gelfand space $\Delta\left(L^{1}(G, \omega)\right)$ is homeomorphic to $H(G, \omega)$ equipped with the compact-open topology.

Proof of Theorem 4.1. The "only if" part is clear.

Conversely, suppose that $L^{1}(G, \omega)$ has UUNP. Fix $\alpha \in H(G, \omega)$. Define $T_{\alpha}: \widehat{G} \rightarrow H(G, \omega), T_{\alpha}(\theta)=\alpha_{\theta}$. Then $T_{\alpha}$ is a one-one, continuous map. Moreover $T_{\alpha}(\widehat{G})$ is closed in $H(G, \omega)$. Next we show that $T_{\alpha}(\widehat{G})=\widehat{G}$. If possible, choose $\beta \in H(G, \omega) \backslash T_{\alpha}(\widehat{G})$. Then $T_{\alpha}(\widehat{G}) \cap T_{\beta}(\widehat{G})$ is empty. Define $p_{\alpha}(f)=\sup \left\{\left|\widehat{f}\left(\alpha_{\theta}\right)\right|: \theta \in \widehat{G}\right\}$ and $p_{\beta}(f)=\sup \left\{\left|\widehat{f}\left(\beta_{\theta}\right)\right|: \theta \in \widehat{G}\right\}$. Then $p_{\alpha}(\cdot)$ and $p_{\beta}(\cdot)$ are uniform norms on $L^{1}(G, \omega)$. Thus $T_{\alpha}(\widehat{G})$ and $T_{\beta}(\widehat{G})$ are closed sets of uniqueness for $L^{1}(G, \omega)$. By [BhDe1, Theorem 2.3], $\partial\left(L^{1}(G, \omega)\right) \subseteq$ $T_{\alpha}(\widehat{G}) \cap T_{\beta}(\widehat{G})$, which is empty. This is a contradiction. Thus $T_{\alpha}(\widehat{G})=$ $\widehat{G}$. Hence $H(G, \omega)=\Delta\left(L^{1}(G, \omega)\right) \cong \widehat{G}$. Since $\partial\left(L^{1}(G, \omega)\right)$ is closed under translation, $\partial\left(L^{1}(G, \omega)\right)=\widehat{G}$. Thus $L^{1}(G, \omega)$ is weakly regular. Now we prove that it is in fact regular. 
Let $F \subset \widehat{G}$ be closed and $\theta \in \widehat{G} \backslash F$. Let $U$ be a symmetric open neighbourhood of the identity in $\widehat{G}$ such that $U^{2} \theta \cap F=\emptyset$. By weak regularity, there exists a non-zero $f \in L^{1}(G, \omega)$ such that $\widehat{f}(\delta)=0(\delta \in U \theta)$. Since $f$ is non-zero, there exists $\theta_{0}$ in $U$ such that $\widehat{f}\left(\theta_{0} \theta\right) \neq 0$. Define $g=f \theta_{0}$. Then $g \in L^{1}(G, \omega), \widehat{g}(\theta)=\widehat{f}\left(\theta_{0} \theta\right) \neq 0$, and $\widehat{g}(\delta)=\widehat{f}\left(\theta_{0} \delta\right)=0$ for all $\delta$ in $F$. Hence $L^{1}(G, \omega)$ is regular.

TheOREM 4.5. Let $L^{1}(G, \omega)$ be semisimple.

(1) There exists a constant $m>0$ such that $\omega(s) \leq \omega(-s)(s \in G)$ iff $L^{1}(G, \omega)$ is a ${ }^{*}$-algebra with the involution $f^{*}(s)=\overline{f(-s)}(s \in G, f \in$ $\left.L^{1}(G, \omega)\right)$. In this case, $\omega(s) \geq 1$ for all $s \in G$.

(2) Suppose that $L^{1}(G, \omega)$ is $a^{*}$-algebra with the above involution. Then $L^{1}(G, \omega)$ is hermitian iff $\Delta\left(L^{1}(G, \omega)\right) \cong \widehat{G}$.

Proof. (1) Suppose that there exists $m>0$ such that $\omega(s) \leq m \omega(-s)$ $(s \in G)$. Then $L^{1}(G, \omega)$ is clearly a *algebra. Conversely, let $L^{1}(G, \omega)$ be a ${ }^{*}$-algebra with the above involution. Since $L^{1}(G, \omega)$ is semisimple, the involution $*$ is continuous. So there exists $m>0$ such that $\left\|f^{*}\right\| \leq m\|f\|$ $\left(f \in L^{1}(G, \omega)\right)$, which implies that $\omega(s) \leq m \omega(-s)$ for almost all $s \in G$. Since $\omega$ is continuous, the inequality holds for all $s \in G$. Finally, for any $n \in \mathbb{N}$ and $s \in G$,

$$
1 \leq \omega(n s) \omega(-n s) \leq m \omega(n s)^{2} \leq m \omega(s)^{2 n} .
$$

Hence $\omega(s) \geq 1(s \in G)$. This proves (1).

(2) Let $L^{1}(G, \omega)$ be hermitian and $\alpha \in H(G, \omega)$. Then for each $f \in$ $L^{1}(G, \omega),\left(f^{*}\right)^{\wedge}(\alpha)=\overline{\widehat{f}(\alpha)}$, which implies that

$$
\int_{G} \overline{f(-s)} \alpha(s) d \lambda(s)=\int_{G} \overline{f(-s)} \overline{\alpha(-s)} d \lambda(s) .
$$

Hence $\alpha(s)=\overline{\alpha(-s)}(s \in G)$ because $\alpha$ is continuous. Now $|\alpha(s)|^{2}=$ $\alpha(s) \overline{\alpha(s)}=\alpha(s) \alpha(-s)=\alpha(0)=1$, and so $|\alpha(s)|=1(s \in G)$. Hence $\alpha \in \widehat{G}$. Thus $\Delta\left(L^{1}(G, \omega)\right)=H(G, \omega) \cong \widehat{G}$. Conversely, suppose that $L^{1}(G, \omega)$ is a Banach *-algebra and $\Delta\left(L^{1}(G, \omega)\right) \cong \widehat{G}$. Then the hermiticity of $L^{1}(G)$ implies that $\left(f^{*}\right)^{\wedge}(\theta)=\overline{\widehat{f}(\theta)}$ for each $\theta \in \widehat{G}$. Hence $L^{1}(G, \omega)$ is hermitian. This proves (2).

Proposition 4.6. Let $H$ be an additive subgroup of the rationals $\mathbb{Q}$ containing 1 . Let $\omega(s) \geq 1$ for all $s$ in $H$. Then $\ell^{1}(H, \omega)$ has UUNP iff $\ell^{1}(\mathbb{Z}, \omega)$ has UUNP.

Proof. By the above, it is sufficient to show that $\ell^{1}(\mathbb{Z}, \omega)$ is regular iff $\ell^{1}(H, \omega)$ is regular. We shall use a result due to Domar [Do] that for an abelian group $G$ with a weight $\omega$ such that $\omega(s) \geq 1$ for all $s \in G, \ell^{1}(G, \omega)$ 
is regular iff it is non-quasi-analytic in the sense that $\sum\left\{\log \omega(n s) / n^{2}\right.$ : $n \in \mathbb{N}\}<\infty$ for all $s \in H$. Assume that $\ell^{1}(\mathbb{Z}, w)$ is regular. Fix $s=p / q \in$ $H^{+} \backslash(0),(p, q)=1$. Then

$$
\begin{aligned}
\sum_{n=1}^{\infty} \frac{\log \omega(n s)}{n^{2}} & =\sum_{j=1}^{q}\left(\sum_{k=0}^{\infty} \frac{\log \omega((k q+j) s)}{(k q+j)^{2}}\right) \\
& \leq \sum_{j=1}^{q}\left(\sum_{k=0}^{\infty} \frac{\log \omega(k p)+\log \omega(j s)}{(k q+j)^{2}}\right) \\
& \leq \sum_{j=1}^{q}\left(\sum_{k=0}^{\infty} \frac{p \log \omega(k)}{(k q+j)^{2}}+\sum_{k=0}^{\infty} \frac{\log \omega(j s)}{(k q+j)^{2}}\right)<\infty .
\end{aligned}
$$

Similarly we can prove that $\sum_{n=1}^{\infty}(\log \omega(n s)) / n^{2}<\infty$ when $s \in H^{-} \backslash(0)$. Thus $\ell^{1}(H, \omega)$ is regular.

The Beurling algebras $L^{1}(G, \omega)$ can also be considered when $G$ is a semigroup. The following illustrates what can happen in this case.

Proposition 4.7. (1) $L^{1}\left(\mathbb{R}^{+}, \omega\right)$ does not have UUNP for any weight $\omega$.

(2) $\ell^{1}\left(\mathbb{Z}^{+}, \omega\right)$ does not have UUNP for any weight $\omega$.

Proof. (1) Let $\sigma=\inf \{\log \omega(s) / s: s>0\}>-\infty$. Now $\Delta\left(L^{1}\left(\mathbb{R}^{+}, \omega\right)\right) \cong$ $\bar{\Pi}_{\sigma}=\{z=x+i y: x \geq \sigma\}$ via Laplace transform, and $L^{1}\left(\mathbb{R}^{+}, \omega\right)$ is identified with a subalgebra of $\mathcal{A}\left(\bar{\Pi}_{\sigma}\right)=\left\{f \in C\left(\bar{\Pi}_{\sigma}\right): f\right.$ is analytic in $\left.\Pi_{\sigma}\right\}$, which obviously does not have UUNP. If $\sigma=-\infty$, then $L^{1}\left(\mathbb{R}^{+}, \omega\right)$ is radical.

(2) Let $\varrho=\inf \left\{\omega(n)^{1 / n}: n>0\right\}>0$. Then $\Delta\left(\ell^{1}\left(\mathbb{Z}^{+}, \omega\right)\right)=D_{\varrho}=\{z \in$ $\mathbb{C}:|z| \leq \varrho\}$, and $\ell^{1}\left(\mathbb{Z}^{+}, \omega\right)$ is identified with a subalgebra of the disc algebra, which does not have UUNP. If $\varrho=0$, then $\ell^{1}\left(\mathbb{Z}^{+}, \omega\right)$ is not semisimple.

5. Uniform algebras on polydiscs and balls in $\mathbb{C}^{n}$. For $z=\left(z_{1}, \ldots\right.$ $\left.\ldots, z_{n}\right) \in \mathbb{C}^{n}$, let $|z|_{\infty}=\max \left(\left|z_{1}\right|, \ldots,\left|z_{n}\right|\right),|z|_{2}=\left(\left|z_{1}\right|^{2}+\ldots+\left|z_{n}\right|^{2}\right)^{1 / 2}$. Let $\Delta^{n}=\left\{z \in \mathbb{C}^{n}:|z|_{\infty}<1\right\}$ be the open polydisc, $B^{n}=\left\{z \in \mathbb{C}^{n}:|z|_{2}<1\right\}$ the open ball. Then the polydisc algebra $A\left(\bar{\Delta}^{n}\right)=\left\{f \in C\left(\bar{\Delta}^{n}\right): f\right.$ is holomorphic in $\left.\Delta^{n}\right\}\left(\bar{\Delta}^{n}=\overline{\Delta^{n}}\right)[\mathrm{R} 1]$, the ball algebra $A\left(\overline{B^{n}}\right)=\left\{f \in C\left(\overline{B^{n}}\right): f\right.$ is holomorphic in $\left.B^{n}\right\}$ [R2], as well as the algebras $H^{\infty}\left(\Delta^{n}\right)$ and $H^{\infty}\left(B^{n}\right)$ of bounded holomorphic functions (all with supnorms on their respective domains) fail to have UUNP. We construct variants of these algebras that have UUNP (in fact, SEP), but are not weakly regular. These will be particular cases of an abstract construction described below.

Let $X$ be a compact Hausdorff space, and let $\mathcal{A}$ be a uniform Banach algebra of continuous functions on $X$. For a closed subset $K$ of $X$, let $\mathcal{I}_{K}=$ $\{f \in C(X): f=0$ on $K\},\left.\mathcal{A}\right|_{K}=\left\{\left.f\right|_{K}: f \in \mathcal{A}\right\}$. Then $\mathcal{A}+\mathcal{I}_{K}=\{f+g:$ $\left.f \in \mathcal{A}, g \in \mathcal{I}_{K}\right\}$ is an algebra of functions on $X$, and its closure in $C(X)$ is 
the $u B$-algebra $\mathcal{B}=\left(\mathcal{A}+\mathcal{I}_{K}\right)^{-}=\left\{f \in C(X):\left.f\right|_{K} \in\left(\left.\mathcal{A}\right|_{K}\right)^{-}\right.$(closure in $C(K))\}$.

Proposition 5.1. Assume that:

(a) $\partial\left(\left(\left.\mathcal{A}\right|_{K}\right)^{-}\right)=$bdry $K$, the topological boundary of $K$.

(b) $K=(\operatorname{int} K)^{-}$.

Then:

(1) $\mathcal{B}$ has SEP, and hence has UUNP;

(2) $\mathcal{B}$ is not weakly regular.

Lemma 5.2. Let $K$ be a closed subset of a compact Hausdorff space $X$. Let $\mathcal{A}$ be a uB-algebra of continuous functions on X. Then:

(1) $\Delta\left(\left(\mathcal{A}+\mathcal{I}_{K}\right)^{-}\right)=X \dot{\cup} \Delta\left(\overline{\left.\mathcal{A}\right|_{K}}\right)(\dot{\cup}$ denotes disjoint union $)$.

(2) $\partial\left(\left(\mathcal{A}+\mathcal{I}_{K}\right)^{-}\right)=(X \backslash K)^{-} \cup \partial\left(\overline{\left.\mathcal{A}\right|_{K}}\right)$.

Proof. The essential set $E(\mathcal{A})$ of $\mathcal{A}$ (defined to be the zero set of the largest ideal of $C(X)$ contained in $\mathcal{A})$ is known to satisfy $E(\mathcal{A})^{\mathrm{c}} \subset \mathrm{Ch}(\mathcal{A})$, $\mathrm{Ch}(\cdot)$ denoting the Choquet boundary. Also, $E\left(\left(\mathcal{A}+\mathcal{I}_{K}\right)^{-}\right) \subset K$. Hence $K^{\mathrm{c}} \subset E\left(\left(\mathcal{A}+\mathcal{I}_{K}\right)^{-}\right)^{\mathrm{c}} \subset \operatorname{Ch}\left(\left(\mathcal{A}+\mathcal{I}_{K}\right)^{-}\right)$. It follows that $\operatorname{Ch}\left(\left(\mathcal{A}+\mathcal{I}_{K}\right)^{-}\right)=$ $\left[\mathrm{Ch}\left(\left(\mathcal{A}+\mathcal{I}_{K}\right)^{-}\right) \cap K\right] \cup K^{\mathrm{c}}$. Also, as $E\left(\left(\mathcal{A}+\mathcal{I}_{K}\right)^{-}\right) \subseteq K, K$ is a peak set in the weak sense for $\left(\mathcal{A}+\mathcal{I}_{K}\right)^{-}$. Hence $\operatorname{Ch}\left(\left(\mathcal{A}+\mathcal{I}_{K}\right)^{-}\right) \cap K=\operatorname{Ch}\left(\left.\left(\mathcal{A}+\mathcal{I}_{K}\right)^{-}\right|_{K}\right)=$ $\mathrm{Ch}\left(\left(\left.\mathcal{A}\right|_{K}\right)^{-}\right)$. It follows that $\mathrm{Ch}\left(\left(\mathcal{A}+\mathcal{I}_{K}\right)^{-}\right)=\operatorname{Ch}\left(\left(\left.\mathcal{A}\right|_{K}\right)^{-}\right) \cup K^{\mathrm{c}}$. Now taking closures, we obtain $\partial\left(\left(\mathcal{A}+\mathcal{I}_{K}\right)^{-}\right)=\partial\left(\left(\left.\mathcal{A}\right|_{K}\right)^{-}\right) \cup\left(K^{\mathrm{c}}\right)^{-}$.

Proof of Proposition 5.1. To prove that $\mathcal{B}$ has SEP, it is sufficient to show, in view of [Me1, Theorem 1], that, given a closed subset $F$ of $\Delta(\mathcal{B})$ not containing $\partial(\mathcal{B})$, there exists a non-zero $f \in \mathcal{B}$ such that $f \equiv 0$ on $F$ but $r_{\mathrm{p}}(f)>0$. Here $r_{\mathrm{p}}(f)$ is the permanent radius $r_{\mathrm{p}}(f)=\inf \{\|f\|:\|\cdot\|$ is any submultiplicative, not necessarily complete, norm on $\mathcal{B}\}$. By $[\mathrm{Me} 1$, Proposition 2], $r_{\mathrm{p}}(f)>1$ follows if there exists a non-zero $g \in \mathcal{B}$ such that $f g=g$. Note that, by (a) and (b) above and Lemma $5.2, \partial(\mathcal{B})=\partial\left(\overline{\left.\mathcal{A}\right|_{K}}\right) \cup$ $\overline{K^{\mathrm{c}}}=\operatorname{bdry} K \cup \overline{K^{\mathrm{c}}},(\partial(\mathcal{B}))^{\mathrm{c}}=(\operatorname{bdry} K)^{\mathrm{c}} \cap\left(\overline{K^{\mathrm{c}}}\right)^{\mathrm{c}}=(\operatorname{bdry} K)^{\mathrm{c}} \cap \operatorname{int} K=$ int $K$, hence $\left((\partial(\mathcal{B}))^{\mathrm{c}}\right)^{-}=K$.

Let $F$ be as above.

CASE (i): $\partial(\mathcal{B}) \cap F \neq \emptyset$. Let $F_{1}=F \cup\left((\partial(\mathcal{B}))^{\mathrm{c}}\right)^{-}$, a closed set. There exists $x \in \Delta(\mathcal{B}) \backslash F_{1}$. There exists a compact neighbourhood $K_{0}$ of $x$ in $\Delta(\mathcal{B})$ such that $K_{0} \subset F_{1}^{c}$. Let $U=\operatorname{int} K_{0}$. By Urysohn's lemma, there exists $f \in C(\Delta(\mathcal{B}))$ such that $0 \leq f \leq 1, f \equiv 1$ on $K_{0}$, and $\operatorname{supp} f \subset F_{1}^{c}$. Since $f \equiv 0$ on $F_{1} \supseteq K, f \in \mathcal{I}_{K}$, and hence $f \in \mathcal{B}$. Further, there exists a non-zero $g \in C(\Delta(\mathcal{B}))$ such that supp $g \subset U$. Hence $g \equiv 0$ on $U^{\mathrm{c}} \supseteq K$, so that $g \in \mathcal{I}_{K} \subset \mathcal{B}$. Clearly $f g=g$. 
CASE (ii): $\partial(\mathcal{B}) \cap F=\emptyset$. Then $F \subset\left((\partial(\mathcal{B}))^{\mathrm{c}}\right)^{-}=K$. There exists a non-empty open set $U$ in $\Delta(\mathcal{B}) \backslash K$ such that $\bar{U} \subset \Delta(\mathcal{B}) \backslash K$, and then there exists $f \in C(\Delta(\mathcal{B}))$ with $f \equiv 0$ on $K$ and $f \equiv 1$ on $U$. Taking $g$ as above, we get $f g=g$. This proves that $\mathcal{B}$ has SEP [Me1, Theorem 1], and hence has UUNP. Since $\partial(\mathcal{B}) \neq \Delta(\mathcal{B}), \mathcal{B}$ is not weakly regular.

Remark 5.3. Let $X=\bar{\Delta}$, and let $0<r<1$. Set $K=\{z \in \bar{\Delta}$ : $r \leq|z| \leq 1\}$. Let $\mathcal{A}=A(\bar{\Delta})$. Take $\mathcal{B}=\left(\mathcal{A}+\mathcal{I}_{K}\right)^{-}=\{f \in C(\bar{\Delta})$ : $\left.\left.f\right|_{K} \in \overline{\left.\mathcal{A}\right|_{K}}\right\}=\left\{f \in C(\bar{\Delta}):\left.\left.f\right|_{K} \in \mathcal{A}\right|_{K}\right\}$. Let $\Delta_{r}=\{z:|z|<r\}$. Then $\partial\left(\overline{\left.\mathcal{A}\right|_{K}}\right)=\partial(\mathcal{A})=\Gamma=\{z:|z|=1\} \neq \operatorname{bdry} K, \partial(\mathcal{B})=\bar{\Delta}_{r} \cup \Gamma, K$ does not satisfy (a) of 5.1, and $\mathcal{B}$ fails to have UUNP. Thus (a) cannot be omitted. On the other hand, take $K$ to be $\Gamma=\{z:|z|=1\}$. Then $\left.\mathcal{A}\right|_{\Gamma}=A(\Gamma)$ is the disc algebra on $\Gamma, \mathcal{B}=A(\bar{\Delta})+\mathcal{I}_{\Gamma}=\left\{f \in C(\bar{\Delta}):\left.f\right|_{\Gamma} \in A(\Gamma)\right\}, \Delta(\mathcal{B})=\bar{\Delta}$, and $\partial(\mathcal{B})=\partial(\mathcal{A}(\Gamma)) \cup \overline{\Gamma^{\mathrm{c}}}=\bar{\Delta}$. Note that (b) of 5.1 is not satisfied. However, if $K_{0}$ is a proper closed subset of $\bar{\Delta}$, then there exists a non-empty, compact set $F$ in $\bar{\Delta}$ such that $F \subset\left(K_{0} \cup \Gamma\right)^{\mathrm{c}}$. By Urysohn's lemma, there exists $f \in C(\Delta)$ such that $f \equiv 1$ on $F$ and supp $f \subset\left(K_{0} \cup \Gamma\right)^{\mathrm{c}}$. Hence $f=0$ on $\Gamma$, so that $f \in \mathcal{I}_{\Gamma} \subset \mathcal{B}$. Thus no proper closed subset of $\bar{\Delta}$ is a set of uniqueness for $\mathcal{B}$. Hence $\mathcal{B}$ has UUNP. This shows that assumption (b) can possibly be improved. However, for the applications that follow, it suffices.

Now let $0<r<1$. Let $\Delta_{r}^{n}=\left\{z \in \mathbb{C}^{n}:|z|_{\infty}<r\right\}, B_{r}^{n}=\left\{z \in \mathbb{C}^{n}:\right.$ $\left.|z|_{2}<r\right\}$. Consider the following uniform algebras:

$$
\begin{aligned}
A_{r}\left(\Delta^{n}\right) & =\left\{f \in C\left(\bar{\Delta}^{n}\right): f \text { is holomorphic in } \Delta_{r}^{n}\right\}, \\
A_{r}\left(B^{n}\right) & =\left\{f \in C\left(\overline{B^{n}}\right): f \text { is holomorphic in } B_{r}^{n}\right\}, \\
H_{r}^{\infty}\left(\Delta^{n}\right) & =\left\{f \in C_{\mathrm{b}}\left(\Delta^{n}\right): f \text { is holomorphic in } \Delta_{r}^{n}\right\}, \\
H_{r}^{\infty}\left(B^{n}\right) & =\left\{f \in C_{\mathrm{b}}\left(B^{n}\right): f \text { is holomorphic in } B_{r}^{n}\right\} .
\end{aligned}
$$

Proposition 5.1 gives the following.

Corollary 5.4. Each of $A_{r}\left(\Delta^{n}\right), A_{r}\left(B^{n}\right), H_{r}^{\infty}\left(\Delta^{n}\right)$ and $H_{r}^{\infty}\left(B^{n}\right)$ has $S E P$ and UUNP, but neither of them is weakly regular.

6. Multipliers and measure algebras. Let $\mathcal{A}$ be a semisimple commutative Banach algebra. A multiplier on $\mathcal{A}$ is a linear map $T: \mathcal{A} \rightarrow \mathcal{A}$ such that $(T x) y=x(T y)=T(x y)(x, y \in \mathcal{A})$. Let $M(\mathcal{A})$ denote the algebra of all (automatically) continuous multipliers on $\mathcal{A}$. Then $M(\mathcal{A})$ is a semisimple commutative Banach algebra with the operator norm $\|T\|=$ $\sup \{\|T x\|:\|x\| \leq 1\}$. For $a \in \mathcal{A}$, let $L_{a}: \mathcal{A} \rightarrow \mathcal{A}$ be the multiplication operator $L_{a}(x)=a x(x \in \mathcal{A})$. Then the mapping $a \mapsto L_{a}$ is a one-one, continuous homomorphism from $\mathcal{A}$ onto the ideal $\left\{L_{a}: a \in \mathcal{A}\right\}$ of $M(\mathcal{A})$. This map is an isometry if $\mathcal{A}$ has a bounded approximate identity, say $\left\{e_{i}\right\}$, satisfying $\left\|e_{i}\right\| \leq 1$ for all $i$. The derived algebra $\mathcal{A}_{0}$ of $\mathcal{A}$ is the subalgebra 
$\mathcal{A}_{0}=\left\{x \in \mathcal{A}: \psi \widehat{x} \in \widehat{\mathcal{A}}\right.$ for all $\left.\psi \in C_{0}(\Delta(\mathcal{A}))\right\}$. If $\mathcal{A}$ is selfadjoint (i.e. having a hermitian involution), then $\mathcal{A}_{0}=\{x \in \mathcal{A}: \sup \{\|x y\|: r(y) \leq 1$, $y \in \mathcal{A}\}<\infty\}$. For multipliers, we refer to [La1].

Theorem 6.1. (1) If $M(\mathcal{A})$ has UUNP, then $\mathcal{A}$ has UUNP.

(2) Let $\mathcal{A}$ be a uB-algebra. Then $\mathcal{A}$ has UUNP iff $M(\mathcal{A})$ has UUNP.

(3) Assume that $\mathcal{A}$ admits a hermitian involution. Then $\mathcal{A}_{0}$ has UUNP. If $\mathcal{A}$ is weakly complete or if $\mathcal{A}_{0}^{2}$ is dense in $\mathcal{A}_{0}$, then $M\left(\mathcal{A}_{0}\right)$ has UUNP.

(4) Let $\mathcal{A}$ be an $H^{*}$-algebra. Then each of $\mathcal{A}, M(\mathcal{A})$ and $K(\mathcal{A})$ (compact multipliers) has UUNP.

(1) above compares with [La1, Theorem 1.4.4] that if $M(\mathcal{A})$ is regular, then $\mathcal{A}$ is regular, but the converse does not hold. We do not know whether there exists a regularity analogue of (2) above.

Proof of Theorem 6.1. (1) Assume $M(\mathcal{A})$ has UUNP. Let $|\cdot|$ be any uniform norm on $\mathcal{A}$. On $M(\mathcal{A})$, define $\mid \cdot{ }_{r}$ by $|T|_{r}=\sup \{|T x|: r(x) \leq 1\}$. Notice that for any $x \in \mathcal{A}, r_{\mathcal{A}}(x)=r_{M(\mathcal{A})}\left(L_{x}\right)=r(x)$ (say). Then $|T x| \leq$ $r(T x)=r\left(T L_{x}\right) \leq r(T) r\left(L_{x}\right)=r(T) r(x)$. Thus $|T|_{r}<\infty$, and $|T x| \leq$ $|T|_{r} r(x)(x \in \mathcal{A}, T \in M(\mathcal{A}))$. Clearly, $|\cdot|_{r}$ is a norm on $M(\mathcal{A})$. Further,

$$
\begin{aligned}
|T|_{r}^{2} & =\sup \left\{|T x|^{2}: r(x) \leq 1, x \in \mathcal{A}\right\}=\sup \left\{\left|(T x)^{2}\right|: r(x) \leq 1, x \in \mathcal{A}\right\} \\
& =\sup \left\{\left|T^{2} x^{2}\right|: r(x) \leq 1, x \in \mathcal{A}\right\}=\sup \left\{\left|T^{2} x\right|: r(x) \leq 1, x \in \mathcal{A}\right\} \\
& =\left|T^{2}\right|_{r}
\end{aligned}
$$

showing that $|\cdot|_{r}$ is a uniform norm. Since $M(\mathcal{A})$ has UUNP, it follows that $|T|_{r}=r(T)$ for all $T \in M(\mathcal{A})$. Then, for any $a \in \mathcal{A},\left|L_{a}\right|_{r}=r\left(L_{a}\right)=r(a) \geq$ $|a|$; whereas $\left|L_{a}\right|_{r}=\sup \{|a x|: r(x) \leq 1\} \leq|a| \sup \{|x|: r(x) \leq 1\} \leq|a|$. Hence $|a|=\left|L_{a}\right|_{r}=r(a)(a \in \mathcal{A})$, showing that $\mathcal{A}$ has UUNP.

(2) By [La1, Theorem 1.4.1, p. 24] every $\varphi \in \Delta(\mathcal{A})$ can be uniquely extended to a $\varphi^{\prime} \in \Delta(M(\mathcal{A}))$. This defines a homeomorphic embedding $\Phi: \varphi \in \Delta(\mathcal{A}) \mapsto \varphi^{\prime} \in \Delta(M(\mathcal{A}))$ for respective Gelfand topologies [La1, Theorem 1.4.2, p. 25] having $\Delta^{1}(\mathcal{A})=\left\{\varphi^{\prime}: \varphi \in \Delta(\mathcal{A})\right\}=\Phi(\Delta(\mathcal{A}))$ open in $\Delta(M(\mathcal{A}))$. Suppose that $\mathcal{A}$ is a $u B$-algebra. By [La1, Corollary 1.4.4, p. 26], $M(\mathcal{A})$ becomes a $u B$-algebra. Let $\partial^{1}(\mathcal{A})=\Phi(\partial(\mathcal{A}))$. By [La1, Theorem 1.5.2, p. 29] the Shilov boundary of $M(\mathcal{A})$ is $\partial(M(\mathcal{A}))=\left(\partial^{1}(\mathcal{A})\right)^{-}$(closure in $\Delta(M(\mathcal{A}))$ ). Let $K \subset \Delta(M(\mathcal{A}))$ be a closed set of uniqueness for $M(\mathcal{A})$. Then $\Phi^{-1}\left(K \cap \Delta^{1}(\mathcal{A})\right)$ is a closed set of uniqueness for $\mathcal{A}$. If $\mathcal{A}$ has UUNP, then $\partial(\mathcal{A}) \subset \Phi^{-1}\left(K \cap \Delta^{1}(\mathcal{A})\right)$. Hence $\partial(M(\mathcal{A}))=\left(\partial^{1}(\mathcal{A})\right)^{-} \subset K$, showing that $\partial(M(\mathcal{A}))$ is the smallest closed set of uniqueness for $M(\mathcal{A})$. By $[\mathrm{BhDe} 1$, Theorem 2.3], $M(\mathcal{A})$ has UUNP.

(3) Assume that $\mathcal{A}$ is also a hermitian Banach *-algebra. Then by [La1, Theorem 1.8.3, p. 47], $\mathcal{A}_{0}$ is semisimple, regular, and hermitian. Hence $\mathcal{A}_{0}$ has UUNP. If $\mathcal{A}$ is weakly complete or if $\mathcal{A}_{0}^{2}$ is dense in $\mathcal{A}_{0}$, then by [La1, The- 
orems 1.8.4 and 1.8.5], $M\left(\mathcal{A}_{0}\right)$ is isomorphic to $C_{\mathrm{b}}\left(\Delta\left(\mathcal{A}_{0}\right)\right)$, hence $M\left(\mathcal{A}_{0}\right)$ has UUNP.

(4) Let $\mathcal{A}$ be an $H^{*}$-algebra. By [La1, Theorem 2.1.1, p. 62], $M(\mathcal{A})$ is isometric to $C_{\mathrm{b}}(\Delta(\mathcal{A}))$ and $K(\mathcal{A})$ is isomorphic to $C_{0}(\Delta(\mathcal{A}))$ by [La1, Theorem 2.2.1, p. 64]. Hence $M(\mathcal{A})$ and $K(\mathcal{A})$ have UUNP. By $(1)$, then $\mathcal{A}$ also has UUNP.

In the remaining part of this section, we identify $\mathcal{A}$ with the ideal $\left\{L_{a}\right.$ : $a \in \mathcal{A}\}$ of $M(\mathcal{A})$, so that $\Delta(\mathcal{A})=\Delta^{1}(\mathcal{A}) \subset \Delta(M(\mathcal{A}))=\Delta(\mathcal{A}) \cup h(\mathcal{A})$, where $h(\mathcal{A})=\{\varphi \in \Delta(M(\mathcal{A})): \varphi(x)=0$ for all $x \in \mathcal{A}\}$.

Proposition 6.2. (1) $|T|_{\infty}=\sup \{|\widehat{T}(\varphi)|: \varphi \in \Delta(\mathcal{A})\} \quad(T \in M(\mathcal{A}))$ defines a uniform seminorm on $M(\mathcal{A})$. The spectral radius $T \mapsto r_{M(\mathcal{A})}(T)$ is the greatest uniform norm on $M(\mathcal{A})$.

(2) Let $\mathcal{A}$ be weakly regular. Then for any uniform norm $|\cdot|$ on $M(\mathcal{A})$, $|\cdot|_{\infty} \leq|\cdot| \leq r(\cdot)$ on $M(\mathcal{A})$.

Proof. (1) is immediate. That $r(\cdot)$ is a norm on $M(\mathcal{A})$ is a consequence of commutativity and semisimplicity of $M(\mathcal{A})$.

(2) Let $|\cdot|$ be any uniform norm on $M(\mathcal{A})$. Then there exists a closed set of uniqueness $F$ in $\Delta(M(\mathcal{A}))$ such that $|T|=|T|_{F}=\sup \{|\varphi(T)|: \varphi \in F\}$ $(T \in M(\mathcal{A}))$. Since the Gelfand topology of $\Delta(\mathcal{A})$ is the inherited topology of $\Delta(M(\mathcal{A})), F_{1}=F \cap \Delta(\mathcal{A})$ is a closed subset of $\Delta(\mathcal{A})$. If $F \neq \Delta(\mathcal{A})$, then by the weak regularity of $\mathcal{A}$, there exists a non-zero $a \in \mathcal{A}$ such that $\widehat{a}\left(F_{1}\right)=(0)$. Since $\Delta(M(\mathcal{A}))=\Delta(\mathcal{A}) \cup h(\mathcal{A})$, we have $\widehat{a}(F)=(0)$. As $F$ is a set of uniqueness, this implies that $a=0$. This contradiction shows that $F_{1}=\Delta(\mathcal{A})$. Thus $\Delta(\mathcal{A}) \subset F$. This gives $|T|_{\infty} \leq|T|(T \in M(\mathcal{A}))$.

It would be interesting to know whether in (2) above, weak regularity can be replaced by UUNP.

COROLlary 6.3. Let $\mathcal{A}$ be weakly regular. If $\Delta(\mathcal{A})$ is a set of uniqueness for $M(\mathcal{A})$, then the seminorm $|\cdot|_{\infty}$ is the smallest uniform norm on $M(\mathcal{A})$.

Following [LN], let $M_{00}(\mathcal{A})=\{T \in M(\mathcal{A}): \widehat{T}=0$ on $h(\mathcal{A})\}=k(h(\mathcal{A}))$, and $M_{0}(\mathcal{A})=\left\{T \in M(\mathcal{A}):\left.\widehat{T}\right|_{\Delta(\mathcal{A})} \in C_{0}(\Delta(\mathcal{A}))\right\}$. Then $M_{00}(\mathcal{A})$ and $M_{0}(\mathcal{A})$ are closed ideals of $M(\mathcal{A})$; and $\mathcal{A} \subset M_{00}(\mathcal{A}) \subset M_{0}(\mathcal{A}) \subset M(\mathcal{A})$.

COROLlary 6.4. Let $\mathcal{A}$ be weakly regular. Then $M_{00}(\mathcal{A})$ has UUNP, but $M_{0}(\mathcal{A})$ need not have UUNP.

Proof. By [LN, Proposition 2.1], for all $T \in M_{00}(\mathcal{A})$, we have $\operatorname{sp}(T)=$ $\widehat{T}(\Delta(\mathcal{A})) \cup(0)=\left(\widehat{T}(\Delta(\mathcal{A}))^{-}\right.$(closure), hence $r(T)=|T|_{\infty}$. If $\mathcal{A}$ is weakly regular, then for any uniform norm $|\cdot|$ on $M_{00}(\mathcal{A}),|T|_{\infty} \leq|T| \leq r(T)$ by an argument as in the proof of Proposition 6.2(2). The remaining assertion follows from the discussion on measure algebras at the end of this section. 
Besides $M_{00}(\mathcal{A})$ and $M_{0}(\mathcal{A})$, other sets of interest in $M(\mathcal{A})$ are the following.

$\operatorname{Reg} M(\mathcal{A})=$ the largest regular closed subalgebra of $M(\mathcal{A}) ;$

$\operatorname{Dec} M(\mathcal{A})=\left\{T \in M(\mathcal{A}): L_{T}\right.$ is a decomposable operator on $\left.M(\mathcal{A})\right\}$;

$D M(\mathcal{A})=\{T \in M(\mathcal{A}): T$ is a decomposable operator on $\mathcal{A}\} ;$

$N_{\sigma} M(\mathcal{A})=\left\{T \in M(\mathcal{A}): \operatorname{sp}(T)=\widehat{T}(\Delta(\mathcal{A}))^{-}\right\}$.

By [LN, Theorem 2.10], $\operatorname{Reg} M(\mathcal{A}) \subset \operatorname{Dec} M(\mathcal{A}) \subset D M(\mathcal{A}) \subset N_{\sigma} M(\mathcal{A})$; and if $\mathcal{A}$ has a bounded approximate identity, then $D M(\mathcal{A})$ is a closed subalgebra of $M(\mathcal{A})$. We shall show that $D M(\mathcal{A})$ need not have UUNP, even if $\mathcal{A}$ is regular. On the other hand, if $\mathcal{A}$ is regular, then $\operatorname{Dec} M(\mathcal{A})$ has UUNP. This follows from the proposition below. Note that $N_{\sigma} M(\mathcal{A})$ is known not to be a subalgebra of $M(\mathcal{A})$.

Proposition 6.5. Let $K$ be a closed subalgebra of $M(\mathcal{A})$. Suppose that $\mathcal{A}$ is weakly regular and $\mathcal{A} \subset K \subset N_{\sigma} M(\mathcal{A})$. Then $K$ has UUNP.

Proof. Let $|\cdot|$ be a uniform norm on $K$. Since $K$ is closed in $M(\mathcal{A})$ and since $K \subseteq N_{\sigma} M(\mathcal{A})$, we have $|T|_{\infty}=r_{M(\mathcal{A})}(T)=r_{K}(T) \geq|T|$ for all $T$ in $K$. On the other hand, as in the proof of Proposition 6.2(2) one can see that $|T|_{\infty} \leq|T|$ for all $T \in K$. It follows that $|\cdot|_{\infty}=|\cdot|$ on $K$.

Let $G$ be a locally compact abelian group. Then the convolution Banach algebra $L^{1}(G)$ is a regular, hermitian, semisimple, commutative, Banach *-algebra with involution $f \mapsto f^{*}, f^{*}(s)=\overline{f(-s)}(s \in G)$. Hence, by Proposition 2.10, $L^{1}(G)$ has both UUNP and $\mathrm{U} C^{*} \mathrm{NP}$ as well as is *-regular. Let $M(G)$ be the measure algebra of all complex regular Borel measures on $G$. It is a unital, semisimple, commutative Banach ${ }^{*}$-algebra with involution $\mu \mapsto \mu^{*}, \mu^{*}(E)=\overline{\mu(-E)}$. By [La1, Theorem 0.1.1], $M\left(L^{1}(G)\right) \cong M(G)$ and $L^{1}(G)$ can be identified with the ideal $M_{\mathrm{a}}(G)$ of measures on $G$ that are absolutely continuous with respect to the Haar measure. Let $\widehat{G}$ denote the dual group of $G$. Then we have $\widehat{G} \cong \Delta\left(L^{1}(G)\right) \subset \Delta(M(G))$.

(1) By the uniqueness theorem of Fourier-Stieltjes transform, $\widehat{G}$ is a set of uniqueness for $M(G)$. By Corollary 6.3, $M(G)$ admits a smallest uniform norm; namely $|\mu|_{\infty}=\sup \{|\widehat{\mu}(\theta)|: \theta \in \widehat{G}\}$. If $G$ is non-discrete, then $|\cdot|_{\infty} \neq r(\cdot)$, in which case, $M(G)$ fails to have UUNP. In fact, it follows from Proposition 2.10, [La1, p. 27] and [A, p. 31] that $M(G)$ has UUNP iff $M(G)$ is hermitian iff $M(G)$ is regular iff $G$ is discrete iff $M(G)=D M(G)$. This shows that the converse of Theorem 6.1(1) is not true.

(2) The ideal $M_{00}\left(L^{1}(G)\right) \cong M_{00}(G):=\{\mu \in M(G): \widehat{\mu}=0$ off $\widehat{G}\}$; whereas $M_{0}\left(L^{1}(G)\right) \cong M_{0}(G):=\left\{\mu \in M(G):\left.\widehat{\mu}\right|_{\widehat{G}} \in C_{0}(\widehat{G})\right\}$. Then $L^{1}(G) \subset M_{00}(G) \subset M_{0}(G) \subset M(G)$. For non-discrete $G, L^{1}(G) \neq M_{00}(G)$ 
[A, p. 31], but it has UUNP due to Corollary 6.4. On the other hand, by [N1, p. 309], $M_{0}(G)$ is not hermitian, and so Proposition 2.10 implies that $M_{0}(G)$ does not have UUNP.

(3) $N_{\sigma} M(G)=\left\{\mu \in M(G): \operatorname{sp}_{M(G)}(\mu)=(\widehat{\mu}(\widehat{G}))^{-}=\widehat{\mu}(\widehat{G}) \cup(0)\right\}$, measures with natural spectra [LN]. By [LN, Theorem 4.1], Dec $M(G) \subset$ $N_{\sigma} M(G)$; and Proposition 6.5 implies that

$\operatorname{Dec} M(G)=\{\mu \in M(G): \nu \in M(G) \rightarrow \mu * \nu \in M(G)$ is decomposable $\}$

$$
=\{\mu \in M(G): \widehat{\mu} \text { is hull-kernel continuous on } \Delta(M(G))\}
$$

has UUNP. By [A, Theorem 2.6], and by [LN, Theorem 2.10], $D M\left(L^{1}(G)\right)=$ $D M(G)=\left\{\mu \in M(G): f \in L^{1}(G) \rightarrow \mu * f \in L^{1}(G)\right.$ is decomposable $\}$ is a closed subalgebra of $M(G)$. Again by Proposition 6.5, $D M(G)$ has UUNP.

(4) Assume that $G$ is compact. Then $L^{2}(G)$ is a regular $H^{*}$-algebra. By Theorem 6.1, $L^{2}(G)$ and $M\left(L^{2}(G)\right)$ have UUNP. However by [A, p. 32], $D\left(M\left(L^{2}(G)\right)\right)=M(G)$, which does not have UUNP unless $G$ is finite. This exhibits that $D M(\mathcal{A})$ need not have UUNP, even if $\mathcal{A}$ is regular. On the other hand Dec $M\left(L^{2}(G)\right)$ always has UUNP.

(5) Let $K$ be a closed ${ }^{*}$-subalgebra of $M(G)$. Further, let $L^{1}(G) \subset K \subset$ $N_{\sigma} M(G)$. Then, by Proposition $6.5, K$ has UUNP. By Proposition 2.10, $K$ has $\mathrm{U} C^{*} \mathrm{NP}$, and is hermitian as well as weakly regular.

Acknowledgements. The authors are grateful to R. D. Mehta for a tip leading to Lemma 5.2 and to K. B. Laursen for making the reprints of his papers available. Thanks are also due to the referee for a careful reading of the manuscript.

\section{References}

[A] E. Albrecht, Decomposable systems of operators in Harmonic Analysis, in: Toeplitz Centennial, I. Gohberg (ed.), Oper. Theory Adv. Appl. 4, Birkhäuser, Basel, 1982, 19-35.

[BaDa] W. G. Bade and H. G. Dales, Uniqueness of complete norm for quotients of Banach function algebras, Studia Math. 106 (1993), 289-302.

[Ba] B. A. Barnes, The properties of ${ }^{*}$-regularity and uniqueness of $C^{*}{ }^{*}$ norm in general $^{*}$-algebras, Trans. Amer. Math. Soc. 279 (1983), 841-859.

[Bh1] S. J. Bhatt, Some remarks on hermitian Banach *-algebras, Indian J. Pure Appl. Math. 26 (1995), 131-142.

[Bh2] - Norm-free topological algebra characterizations of $C^{*}$-algebras and uniform Banach algebras, Bull. Polish Acad. Sci. Math. 45 (1997), 117-122.

[BhDe1] S. J. Bhatt and H. V. Dedania, Banach algebras with unique uniform norm, Proc. Amer. Math. Soc. 124 (1996), 579-584.

[BhDe2] - - - Uniqueness of the uniform norm and adjoining identity in Banach algebras, Proc. Indian Acad. Sci. Math. Sci. 105 (1995), 405-409. 
[BD] F. F. Bonsall and J. Duncan, Complete Normed Algebras, Springer, Berlin, 1973.

[DL] H. G. Dales and R. J. Loy, Uniqueness of the norm topology for Banach algebras with finite dimensional radical, Proc. London Math. Soc. (3) 74 (1997), 633661.

[De] H. V. Dedania, A seminorm with square property is automatically submultiplicative, Proc. Indian Acad. Sci. Math. Sci. 108 (1998), 51-53.

[Do] Y. Domar, Harmonic analysis based on certain commutative Banach algebras, Acta Math. 96 (1956), 1-66.

[HKV] W. Hauenschild, E. Kenuith and A. Voigt, ${ }^{*}$-regularity and uniqueness of $C^{*}$ norm for tensor product of ${ }^{*}$-algebras, J. Funct. Anal. 89 (1990), 137-149.

[KN] R. Kantrowitz and M. M. Neumann, On certain Banach algebras of vectorvalued functions, in: Function Spaces (Edwardsville, IL, 1994), K. Jarosz (ed.), Dekker, New York 1995, 223-241.

[La1] R. Larsen, An Introduction to the Theory of Multipliers, Springer, Berlin, 1971.

[La2] -, Banach Algebras: an Introduction, Dekker, New York, 1973.

[LN] K. B. Laursen and M. M. Neumann, Decomposable multipliers and applications to harmonic analysis, Studia Math. 101 (1992), 193-214.

[Me1] M. J. Meyer, The spectral extension property and extension of multiplicative linear functionals, Proc. Amer. Math. Soc. 112 (1991), 855-861.

[Me2] -, Minimal incomplete norms on Banach algebras, Studia Math. 102 (1992), $77-85$.

[N1] M. M. Neumann, Banach algebras, decomposable convolution operators, and a spectral mapping property, in: Function Spaces (Edwardsville, IL, 1990),

K. Jarosz (ed.), Dekker, New York, 1992, 307-323.

[N2] - Commutative Banach algebras and decomposable operators, Monatsh. Math. 113 (1992), 227-243.

[P] T. W. Palmer, Banach Algebras and General Theory of ${ }^{*}$-Algebras, Vol. 2, Cambridge Univ. Press, 2001.

[Ri] C. E. Rickart, General Theory of Banach Algebras, Van Nostrand, 1960.

[R1] W. Rudin, Function Theory in Polydiscs, W. A. Benjamin, 1969.

[R2] - Function Theory in the Unit Ball of $\mathbb{C}^{n}$, Springer, Berlin, 1980.

[TY $] \quad$ B. J. Tomiuk and B. Yood, Incomplete normed algebra norms on Banach algebras, Studia Math. 95 (1989), 119-132.

Department of Mathematics

Sardar Patel University

Vallabh Vidyanagar 388120, India

E-mail: sjb@spu.ernet.in

haresh@spu.ernet.in

Received August 10, 1999

Revised version March 12, 2001 